STOCK OF PLANTS

in Potting You

$$
4,40-41
$$


The following is published for general information:-

\section{LISTS OF PLANTS}

which may usually be obtained from the Botanic Gardens at Singapore and Penang.

(S.) and (P.) mean at Singapore or at Penang only.

* Means suitable for hedge-making.

Unless stated otherwise, the prices are for small plants in pots and the price may be altered according to the size of the plant. Rooted cuttings dug from the nursery and not in pots are charged at 20 to 40 cents, according to size. The cost of packing and all subsequent charges are extra.

Plants not included in the following lists are sometimes available.

\section{FLOWERING TREES}

Bauhinia Blakeana

Bauhinia monandra

Bauhinia purpurea

Brownea ariza (S.)

Brownea grandiceps (S.) . .

Cassia fistula-Indian Laburnum

Cassia grandis-Pink Cassia

Cassia javanica ..

Cassia nodosa-Pink Cassia

Cassia renigera-Burmese Cassia

Cassia multijuga

Cassia siamea-Johar:

Cassia spectabilis

Citharexylum quadrangulare

Cochlospermum religiosum-Buttercup Tree

Cratoxylon formosum

Cratoxylon ligustrinum (P.)

Delonix regia-Flame of the Forest ..

Dillenia indica . .

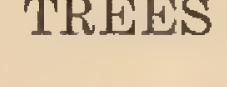

Enterolobium saman-Rain Tree

.

Erythrina indica-Coral Tree (Dadap)

Jacaranda filicifolia

Kigelia pinnata (P.)

Lagerstroemia floribunda (P.)

Lagerstroemia flos-reginae-Rose of India

Lagerstroemia Loudonii

Mesua ferrea-Ceylon Ironwood

Peltophorum pterocarpum-Yellow Flame

Pterocarpus indicus-Angsana

Saraca declinata (S.)

Saraca indica (S.)

Saraca taipingensis (S.)

Spathodea campanulata-African Tulip Tree

Wormia suffruticosa

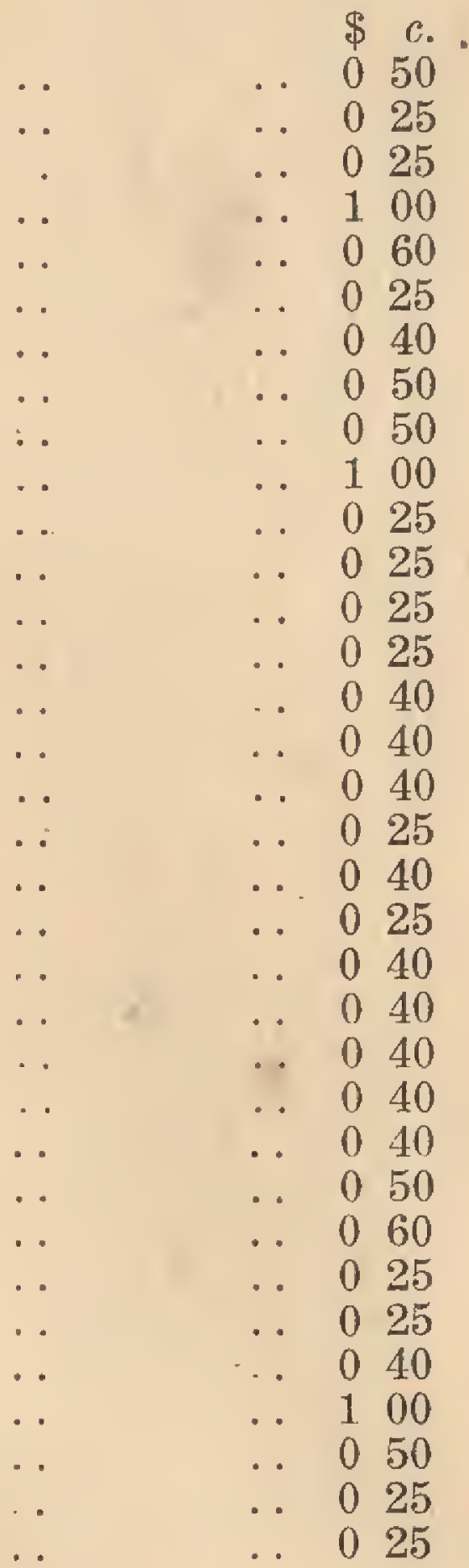


FOLIAGE TREES

Acacia auriculaeformis

Adenanthera pavonina-Saga Tree

Albizzia falcata-Batai

Andira inermis (S.)

Andira surinamensis (S.) ...

Arfeuillea arborescens

Calophyllum inophyllum-Penaga Laut

Canangium odoratum-Kenanga

* Casuarina equisetifolia-Common Ru . .

.

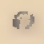

Casuarina rumphiana (S.)

*Casuarina sumatrana (S.) . .

* Cinnamomim iners-Wild Cinnamon

Dacrydium elatum-Ru Bukit

Elaeocarpus ganitrus

Eugenia densiflora (P.)

Eugenia grandis-_Jambu Laut

Fugenia Helferi (P.)

Eugenia longiflora-Kelat ..

Fagraea fragrans-Tembusu

Ficus irregularis

Filicium decipiens (P.)

Gliricidia sepium-Mexican Lilac

Gnetum Gnemon--Meninjau (P.)

Hymenaea courbaril (S.) . .

Melia excelsa (P.)

Millettia atropurpurea (S.)

Muntingia calabura-Cherry Tree

Parkia speciosa (P.) -Petai

* Pithecellobium dulce-Madras Thorn .

*Podocarpus gracilior

* Podocarpus polystachyus

Podocarpus Rumiphii

Polyalthia longifolia (P. . . .

Sapium jamaicense

Sterculia macrophylla (P.) . .

*Streblus asper-per cutting

Swietenia macrophylla_-Broad leaved Mahogany ..

Tamarindus indica-Tamarind

Terminalia catappa-Bengal Almond

\section{SHRUBS}

* Acalypha siamensis

* Acalypha, various species-Cat's Tails .

Allamanda nerififolia

*Allamanda Schottii

Allamanda violacea (P.) ..

* Aliamanda Williamsii

Bauhinia acuminata (white)

Bauhinia tomentosa

* Bixa orellana-Anatto (Kesumba)

Bougainvillea Calcutta No. 2 (S.)

Bougainvillea Calcutta No. 13 (S.)

Bougainvillea Cypheri (S.)

Bougainvillea formosa

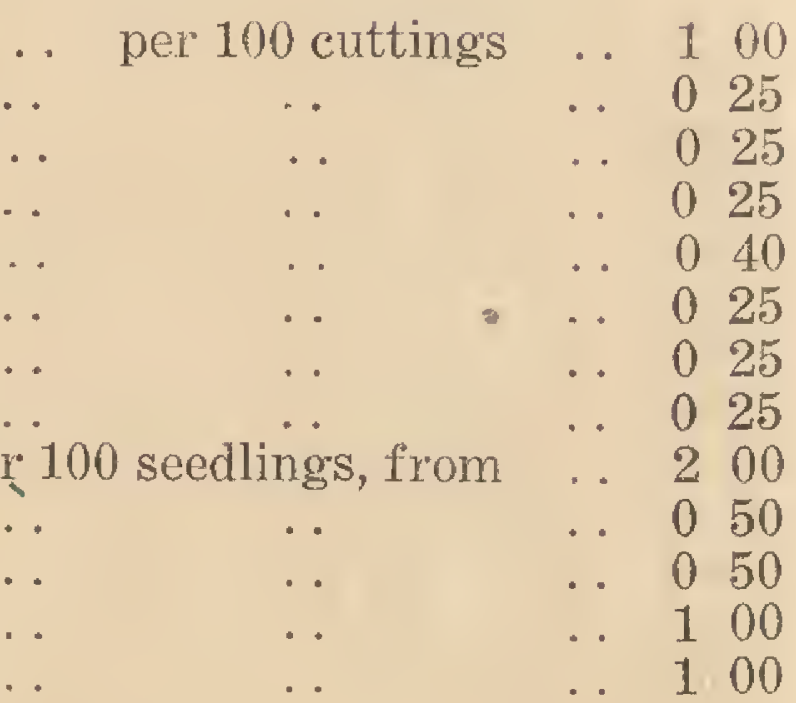




\section{SHRUBS.-continued}

Bougainvillea glabra, pale rose variety

Bougainvillea glabra, var. magnifica.

*Bougainvillea glabra, var. Sanderiana

*Bougainvillea glabra, var. Sanderiana

* Bougainvillea lateritia

*Bougainvillea Mrs. Butt

Bougainvillea Mrs. Fraser . .

Bougainvillea Mrs. Lancaster

*Bougainvillea Mrs. McLean . .

*Bougainvillea Rosa Catalina

Bougainvillea Thomasii

Brunfelsia americana

Brunfelsia eximia (blue)

Brunfelsia undulata (white, large flowers)

Caesalpinia pulcherrima-Peacock Flower

Cáesalpinia pulcherrima var. lutea

Calliandra haematocephala (P.)

Cassia auriculata

Cassia bicapsularis

Cassia biflora

Cassia fruticosa

Cassia splendida

Catesbaea spinosa-Lily Thorn

Clerodendron macrosiphon

Clerodendron ugandense (S.)

* Crotons (Codiaeum) - several races

Cyanophyllum magnificum

*Duranta Ellisii

* Duranta repens

Ehretia microphylla-per cutting

Eranthemum malaccense

Ervatamia divaricata-Susun Kelapa

Euphorbia pulcherrima-Poinsettia .

* Excoecaria 'bicolor

Gardenia florida-Bunga China

Gustavia gracillima

Gustavia speciosa

Hamelia patens (S.)

Hibiscus mutabilis

*Hibiscus rosa-sinensis, various races

Hibiscus rosa-sinensis

Hibiscus, standard plants ..

*Hibiscus schizopetalus

Hibiscus sp. (small pink flowers)

Holmskioldia sanguinea

Honckenya ficifolia

Ipomoea carnea

Ixora chinensis

Ixora coccinea and varieties

Ixora congesta

Ixora finlaysoniana

Ixora javanica and varieties

Ixora nigricans . .

Jasminum bifarium

$$
\begin{aligned}
& \$ c . \\
& \begin{array}{llll}
\ldots & \ldots & 075
\end{array} \\
& \text {. } \quad \ldots 100 \\
& \text {.. } \quad \ldots \quad 025 \\
& \text { per } 100 \text { cuttings .. } 200 \\
& \text {.. } 050 \\
& \text {.. } 025 \\
& \text {. } 075 \\
& \text {.. } 100 \\
& \text {.. } 040 \\
& \text {.. } 050 \\
& \text {.. } 050 \\
& \text {.. } 040 \\
& \therefore \quad 040 \\
& \text {. } 040 \\
& \text {.. } 020 \\
& \text {.. } 020 \\
& \text {. } 040 \\
& \text {. } 025 \\
& \text {. } 025 \\
& \text {. } 025 \\
& \text {. } 025 \\
& \text {.. } 040 \\
& \text {.. } 040 \\
& \text {.. } 025 \\
& \text {.. } 025
\end{aligned}
$$

. 25 cents to 050

per 100 cuttings .. 100

75 cents to 100

. 025

. 025

.. 025

.. 025

.. 025

. 040

.. 040

. 040

. 040

.. 040

. 040

.. 025 


\section{SHRUBS.-continued}

Jasminum glandulosum

Jasminum pubescens

Jasminum sambac

Jatropha multifida

. 025

. . 025

. 025

. 025

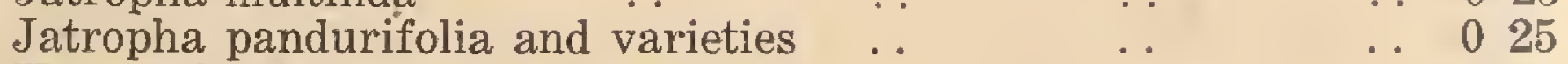

Kopsia fruticosa

Lagerstroemia indica (P.)

Lantana camara, varieties

Malpighia coccigera

*Malpighia nitida

Malvaviscus arboreus (S.).

Malvaviscus Conzattii

Malvaviscus Conzattii Pink .

Manihot utilissima variegata-Variegated Tapioca .

Muehlenbeckia platyclada

Murraya paniculata-Kemuning

Mussaenda erythrophylla . .

Mussaenda luteola

Nerium oleander-Oleander

Ochna pumila (S.)

Panax Guilfoylei

Panax fruticosum

Petrea rugosa (P.)

Phaleria Blumei

Plumeria acutifolia-Frangipanni

Plumeria obtusifolia

Plumeria rubra (Red Frangipanni) and varieties..

Quassia amara-Bitter Quassia

Randia macrantha-Angels Trumpet ..

*Ravenia spectabilis (S.)

Rondeletia odorata

Sambucus javanica-Javanese Elder

Sambucus velutina

Solanum Wrightii-Potato Tree

Stenolobium stans--Yellow Bells

Tecoma Smithii (P.)

*Tecomaria capensis

Thevetia peruviana-yellow, white and apricot

Thunbergia affinis

Thunbergia erecta

Thunbergia Kirkii

Thunbergia Vogeliana

Tithonia diversifolia

Turnera aurantiaca

Uroskinnera spectabilis

$\begin{array}{ll}\ldots & \cdots \\ \therefore & \cdots \\ \therefore & \cdots \\ \therefore & \cdots \\ \cdots & \cdots\end{array}$

.. 025

.. 040

. 020

.. 025

.. 025

.. 025

. 025

. 100

. 050

$\begin{array}{lll}. . & 0 & 40\end{array}$

. 050

. 040

.. 025

. 040

.. 040

. 025

.. 025

.. 040

.. 060

.. 025

. 040

. 040

.. 025

.. 025

. 025

.. 060

.. 025

.. 0225

.. 0225

. 025

. 025

. 025

. 025

. 040

. 025

. 040

.. 025

. 010

.. 025

.. 025

\section{CLIMBERS}

Afgekia sericea

..

Allamanda cathartica

Antigonum leptopus--Honolulu Creeper

Antigonum leptopus-var. album (white)

Aristolochia grandiflora (S.)

Bauhinia Kockiana

Beaumontia multiflora

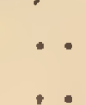

..

$\begin{array}{llll}\ldots & \ldots & 0 & 50 \\ \ldots & \therefore & 0 & 25 \\ \ldots & \cdots & 0 & 25 \\ \cdots & \cdots & 0 & 25 \\ \ldots & \ldots & 0 & 50 \\ \cdots & \ldots & 1 & 00 \\ \cdots & & 0 & 50\end{array}$




\section{CLIMBERS.-continued}

Bignonia magnifica-Purple Bignonia . .

Bignonia argyreo-violascens (S.) ..

Bignonia unguis-cati

Camoensia maxima

Chonemorpha spp.

Clematis triloba-Indian Traveller's Joy

Clerodendron splendens . .

Clerodendron Thomsonae . . .

Clitorea ternatea

Congea tomentosa

.

Congea velutina

Cryptostegia madagascariensis (P.) ..

Faradaya splendida

Ficus pumila (ivy-like creeper) Creeping Fig

Gloriosa superba

Gloriosa virescens

Hosea Lobbii (S.)

(S.) $\quad \cdots \quad \cdots$

Ipomoea digitata-Morning Glory (mauve)

Ipomoea Horsfalliæ, Briggsii

Ipomoea Learii-Morning Glory (blue)

Ipomoea pulchella

Jacquemontia violacea $\quad \cdots$

Jasminum rex ..

Lonicera japonica-Japanese Honeysuckle

Lonicera macrantha-Indian Honeysuckle

Odontadenia speciosa

Pandorea jasminoides

Pandorea pandorana

Passiflora laurifolia-Buah Susu

Passiflora quadrangularis-Grenadilla

Passiflora quadriglandulosa-Pink Passion Flower

Petrea volubilis

Porana volubilis-Bridal Creeper

Quisqualis indica-Drunken Sailor

Roupellia grata

Stephanotis foribunda

Stigmaphyllon ciliatum

Stigmaphyllon lancifolium

Stigmaphyllon sagraeanum

Thunbergia grandiflora

Thunbergia grandiflora var. alba

Thunbergia laurifolia

Tristellateia australasica

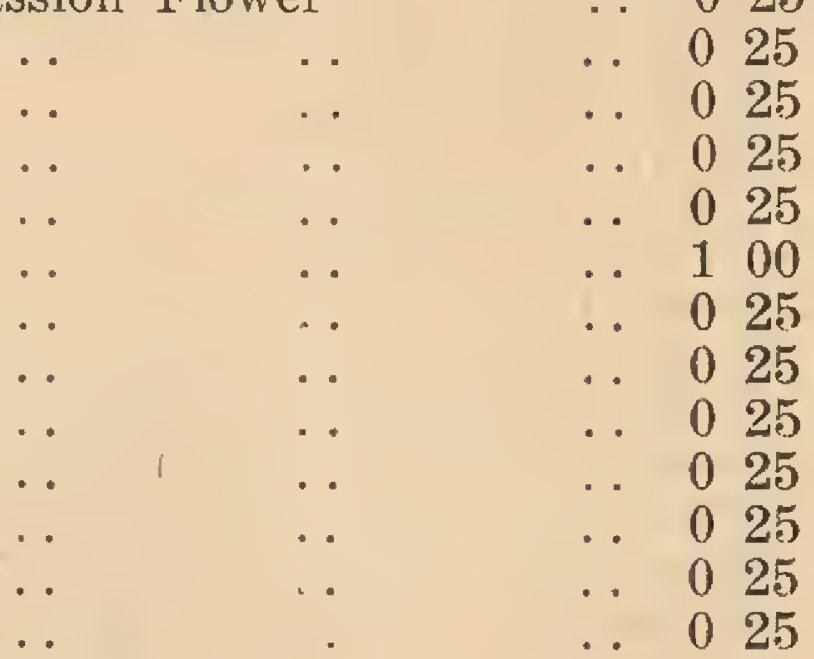

PALMS (in 8 ins. pots)

Acoelorraphe Wrightii (S.)

Actinorhytis calapparia

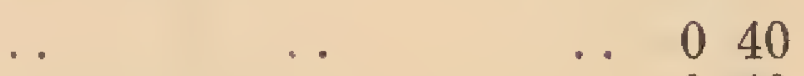

Archontophoenix Alexandrae

$0 \quad 40$

Areca catechu-Betel-nut Palm

$\begin{array}{lllll} & \ldots & \ldots & 0 & 40\end{array}$

Arenga undulifiolia (S.) ..

Carludovica palmata-Panama Hat Palm (Cyclanthacere) $\cdots \quad \begin{array}{lll}4 & 0\end{array}$

Chrysalidocarpus lutescens. . 
PALMS (in 8 ins. pots).-continued

\begin{tabular}{|c|c|c|c|c|}
\hline \multirow{2}{*}{\multicolumn{4}{|c|}{$\begin{array}{l}\text { Cyrtostachys lakka_-Sealing Wax Palm } \\
\text { Elaeis guineensis-African Oil Palm (for ornamental purposes) }\end{array}$}} & \\
\hline & & & & \\
\hline Heterospathe salomonensis (S.) & & . & & \\
\hline Hyophorbe Verschaffeltii (S.) & . & . & & \\
\hline ala grandis & & . & & \\
\hline oninoso & & . & & \\
\hline tona chinensis & & . & . & \\
\hline ona cochin-chinensis & . & . & . & \\
\hline ona rotundifolia & . & . & . & \\
\hline a Wo & . & .. & & \\
\hline perma tigillaria_Nibong & . & . & . & \\
\hline eodoxa oleracea-Cabbage Palm & . & . & & \\
\hline a-Royal Palm & & . & & \\
\hline ga Kuhlii $\quad$.. & . & .. & . & \\
\hline & & . & & \\
\hline McArthuri & & . & . & \\
\hline & & . & & \\
\hline flabelliformis-suckers, not & pots & & & \\
\hline $\operatorname{dra}(\mathrm{S})$. & $\ldots$ & . & .. & \\
\hline & & . & & \\
\hline schaffeltia splendida & & .. & & \\
\hline
\end{tabular}

\section{HERBACEOUS PLANTS AND DWARF SHRUBS}

Ageratum, dwarf

Alternanthera paronichioides

Angelonia salicariaefolia

Artemisia lactiflora

Aster bellidioides

Aster sp. (dwarf)

Aster, perennial-Michaelmas Daisy

Asystasia intrusa

Barleria cristata, four varieties

Barleria lupulina

Beloperone guttata

Canna, hybrids, various races, roots ..

Cestrum nocturnum

Clerodendron paniculatum-Pagoda Flower

Coleus Blumei, various races

Coreopsis grandiflora (perennial)

Crinum asiaticum-Crinum Lily

Crinum giganteum-Java Lily

Crossandra nilotica (P.)

Crossandra undulifolia-Singapore Geranium

Crossandra undulifolia-yellow variety

Cuphea platycentra (P.) ..

Cuphea sp.

Daedalacanthus nervosus . .

Eranthemum atropurpureum

Eranthemum hypocrateriforme

Eranthemum reticulatum

Eranthemum Wattii

Eucharis grandiflora-Amazon Lily

Galphimia glauca

Hedychium coronarium

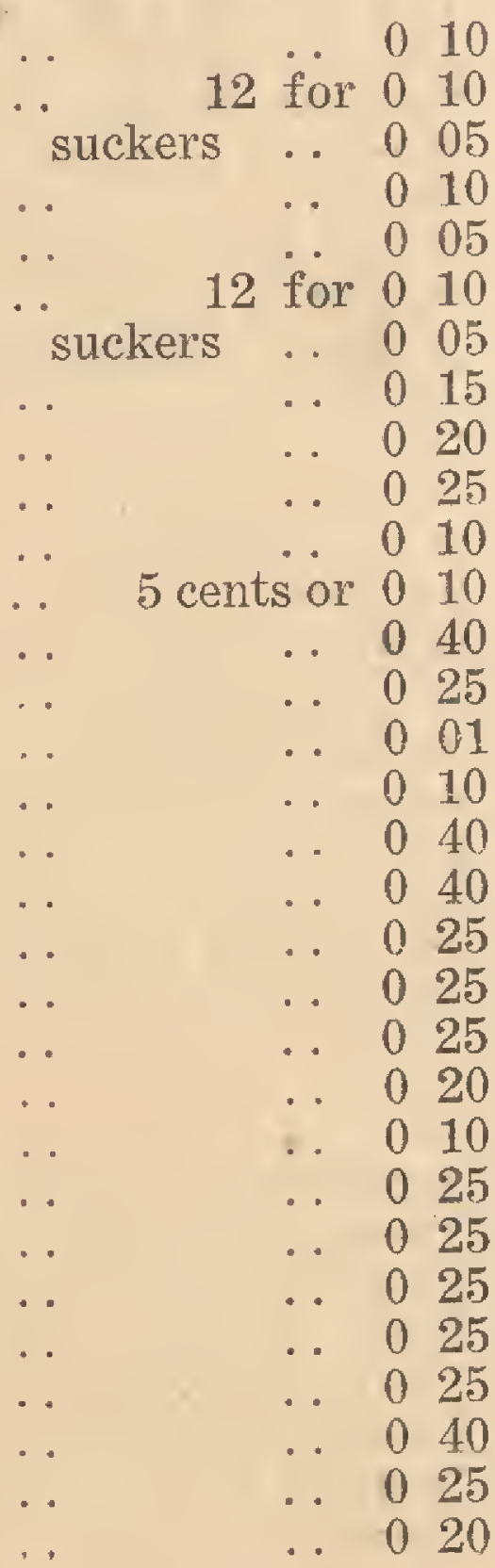


HERBAEEOUS PLANTS AND DWARF SHRUBS.-continued

Helianthus angustifolius-Perennial Sunflower . . $\quad \ldots 005$

Heliconia psittacorum-"Japanese Canna" per root .. 005

Hippeastrum .. $\quad \ldots$. . $\quad \ldots$ bulbs $\ldots 020$

Hymenocallis littoralis-Spider Lily . . $\quad \ldots$ bulbs $\quad \ldots \quad 010$

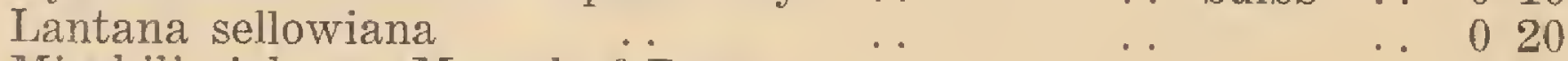

Mirabilis jalapa-Marvel of Peru $\quad \ldots \quad \ldots \quad \ldots 020$

Orthosiphon stamineus-Cat's Whiskers $\quad \ldots \quad \ldots 025$

Oxalis dispar

Pancratium zeylanicum

Pennisetum macrostachyum (S.) ‥

Pentas alba $\ldots \quad \ldots$

Pentas carnea . . $\quad \ldots . \quad \ldots$

Pentas coccinea .. $\quad$. . .

Platycodon grandiflorum . Plumbago ..

Plumbago rosea-Pink Plumbago ..

Pudbeckia Newmanii-Black-Eyed Susan

Ruellia macrophylla

Ruellia malacosperma

Ruellia tuberosa

Russellia juncea

Russellia sarmentosa

Russellia hybrid

Sanchezia nobilis

Turnera ulmifolia

Turnera trioniflora

Tussacia pulchella

Verbena tenera . .

Vinca rosea-Periwinkle

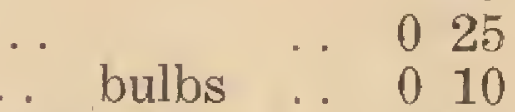

per stool .. $\quad 005$

.. $\quad \ldots \quad 020$

.. $\quad \ldots 020$

. $\quad \ldots 025$

.. $\quad \ldots 025$

. $\quad \ldots 050$

. $\quad \ldots 025$

. $\quad \ldots \quad 010$

.. $\quad \ldots 025$

. $\quad \ldots 025$

. $\quad \ldots 005$

. $\quad \ldots 020$

$\therefore \quad \ldots 020$

. $\quad \ldots 020$

. $\quad \ldots 040$

. $\quad \ldots 020$

. $\quad \ldots 010$

. $\quad \ldots 025$

$\begin{array}{llll}. & \ldots & 0 & 05\end{array}$

- $\quad 010$

Zephyranthes, white, yellow and pink $\cdots \quad$ per bulb $\ldots 0010$

Zephyranthes, hybrids and large flowered forms .. $\quad \ldots 005$

\section{SEEDS OF HERBACEOUS PLANTS}

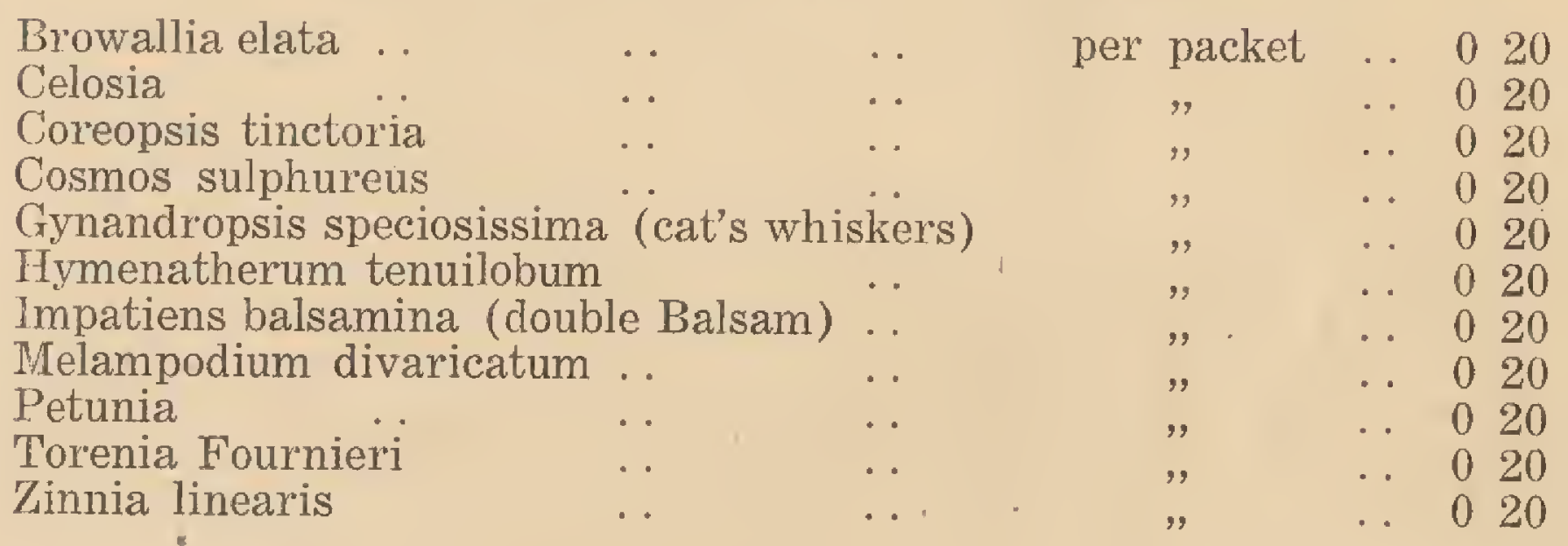

WATER GARDEN PLANTS

Acorus calamus-Sweet Flag (S.) _. $\quad \ldots \quad \ldots \quad \ldots 25$

$\begin{array}{lllll}\text { Cyperus alternifolius } & \ldots & \ldots & \ldots & \\ \text { Cyperus papyrus } & \ldots & \ldots & \ldots & 025\end{array}$

Cyperus papyrus--Nile Papyrus $\quad \ldots \quad \ldots \quad \ldots 025$

Eichhornia crassipes-Water Hyacinth $\quad \cdots \quad \ldots 00$ 
WATER GARDEN PLANTS.-continued

Hydrocleis nymphoides-Watex Poppy

Limnocharis Plumieri

Monochorea elata

Monochorea vaginalis

Montrichardia aculeata

Nelumbium speciosum-Indian Lotus .

Nymphaea in variety-Water Lilies.

Pontederia sp.

Sagittaria montevidensis

Sagittaria sagittifolia

Scirpus mucronatus

$\begin{array}{cccc} & & \$ & c . \\ \ldots & \ldots & 0 & 25 \\ \ldots & \ldots & 0 & 25 \\ \ldots & \ldots & 0 & 25 \\ \ldots & \ldots & 0 & 25 \\ \ldots & \ldots & 0 & 25 \\ \ldots & \ldots & 0 & 25 \\ \text { young plants } & \ldots & 0 & 40 \\ \ldots & \ldots & 0 & 25 \\ \ldots & \ldots & 0 & 25 \\ \ldots & \ldots & 0 & 25 \\ \ldots & \ldots & 0 & 25\end{array}$

\section{ORCHIDS}

Arachnis flos-aeris-Scorpion Orchid cuttings . 0020

Arachnis Hookeriana (S.) - White Scorpion Orchid " " . 020

Arachnis Maingayi-Scorpion Orchid

Vanda Hookeriana

Vanda hybrid Miss Joaquim

Vanda teres

Vanda teres var. Andersonii

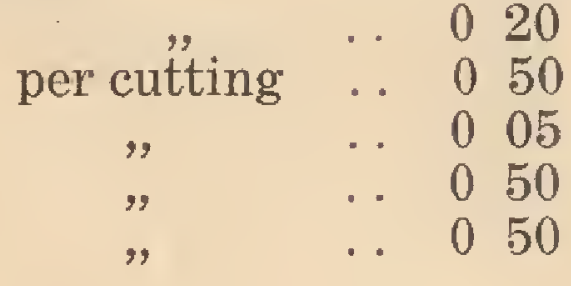

Occasionally plants of other orchids are for sale; prices on application.

[No. C.S.O. $768 / 38$ ] 
(Vide Supplement No. 39 to Gazette of 26th-April, 1940)

The following is published for general information:-

$$
\text { LIST OF PLANTS }
$$

which may usually be obtained from the Botanic Gardens at Singapore and Penang.

(S.) and (P.) mean at Singapore or at Penang only.

* Means suitable for hedge-making.

Unless stated otherwise, the prices are for small plants in pots and the price may be altered according to the size of the plant. Rooted cuttings dug from the nursery and not in pots are charged at 20 . to 40 cents, according to size. The cost of packing and all subsequent charges are extra.

Plants not included in the following lists are sometimes available.

\section{FLOWERING TREES}

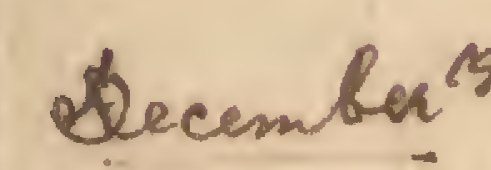

Bauhinia Blakeana

12 Bauhinia monandra

as Bauhinia purpurea

17 Brownea ariza (S.)

2 Brownea grandiceps (S.)

Cassia fistula-Indian Laburnum

Cassia grandis-Pink Cassia

20 Cassia javanica.

Cassia nodosa-Pink Cassia

10 Cassia renigera-Burmese Cassia

Cassia multijuga

4 Cassia siamea-Johar

Cassia spectabilis

Citharexylum quadrangulare

2 Cochlospermum religiosum-Buttercup Tree

Cratoxylon formosum

Cratoxylon ligustrinum (P.)

-Delonix regia-Flame of the Forest

$\mathrm{s}^{-}$Dillenia indica.

65: Enterolobium saman-Rain Tre

Erythrina indica-Coral Tree (Dadap) (P.)

100 Jacaranda filicifolia

Kigelia pinnata (P.)

Lagerstroemia floribunda $\left(P_{.}\right)$.

..

6o Lagerstroemia flos-reginae-Rose of India

10 Lagerstroemia Loudonii

15. Mesua ferrea-Ceylon Ironwood

$\cdots$

10 Peltophorum pterocarpum-Yellow Flame

30 Pterocarpus indicus-Angsana

10 Saraca declinata (S.)

4 Saraca indica (S.)

6 Saraca taipingensis (S.)

35- Spathodea campanulata-African Tulip Tree

4. Wormia suffruticosa 
FOLIAGE TREES

Acacia auriculaeformis

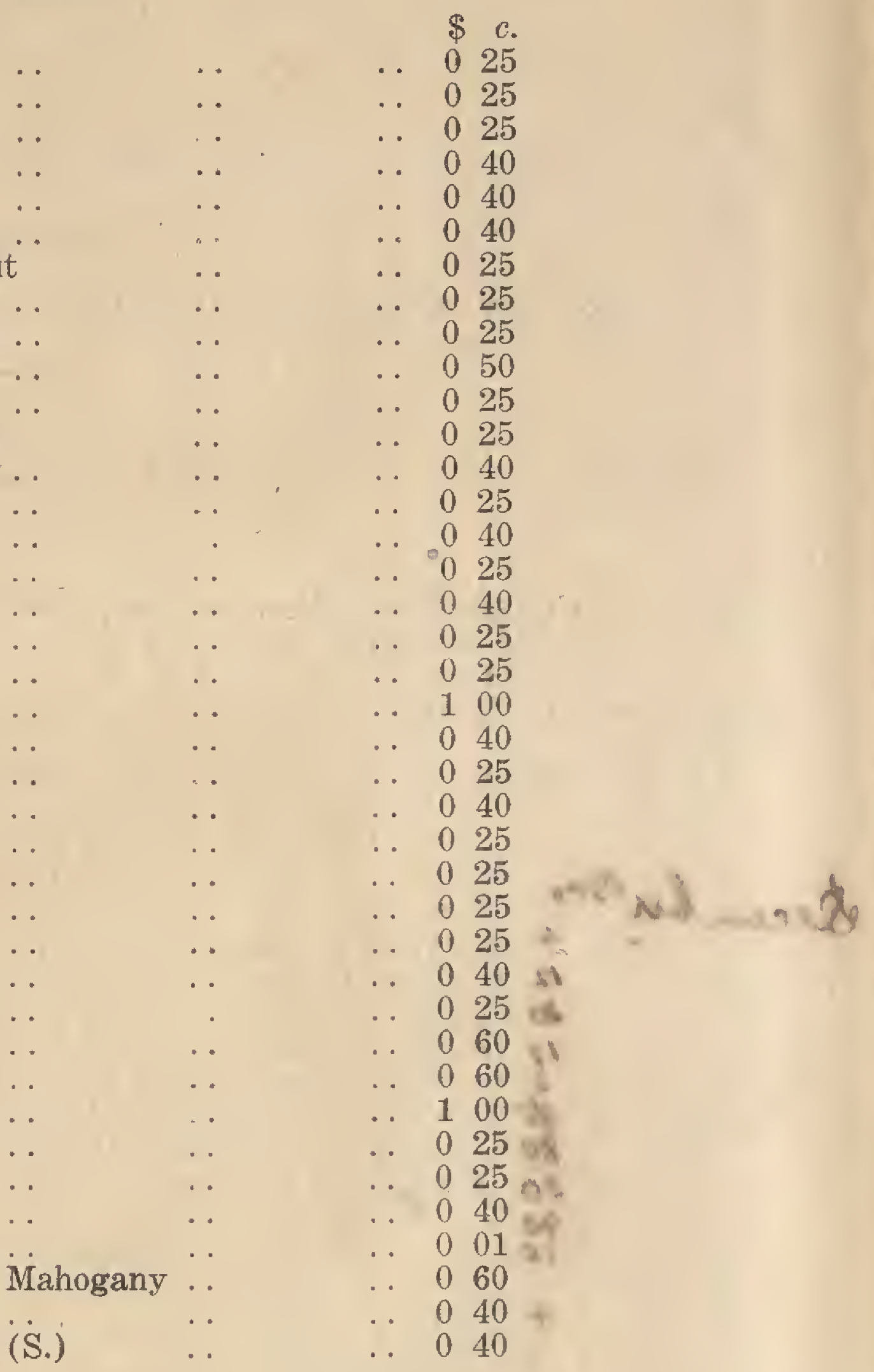

\section{SHRUBS}

$40 *$ Acalypha, various species-Cat's Tails . Allamanda neriifolia

* Allamanda Schottii

Allamanda violacea (P.)

* Allamanda Williamsii

15- Bauhinia acuminata (white)

10 Bauhinia tomentosa

Bixa orellana-Anatto (Kesumba)

35- Bougainvillea Calcutta No. 2.(S.)

13 Bougainvillea Calcutta No. 13 (S.)

30 Bougainvillea Cypheri (S.)

2 Bougainvillea formosa

20 Bougainvillea glabra, pale rose variety

Bougainvillea glabra, var. magnifica

Bougainvillea glabra, var. Sanderiana

10. Bougainvillea lateritia

20 *Bougainvillea Mrs. Butt

20 Bougainvillea Mrs. Fraser .

Bougainvillea Mrs. Lancaster

35*:Bougainvillea Mrs. McLean . .

35 - Bougainvillea Rosa Catalina

15- Bougainvillea Thomasii

10 Brunfelsia americana

o Brunfelsia eximia (blue)

7 Brunfelsia undulata (white, large flowers)

10 Caesalpinia pulcherrima-Peacock Flower

Caesalpinia pulcherrima var. lutea

5 Calliandra haematocephala (P.)

Cassia auriculata

5 Cassia bicapsularis

3 Cassia biflora

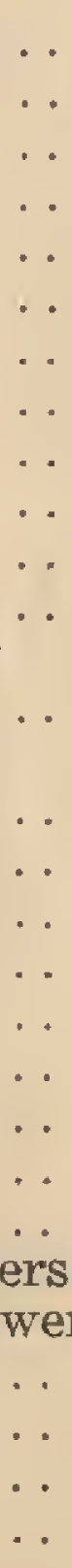

.. $\quad$. 0025




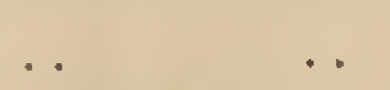

to Cassia splendida

...

4. Catesbaea spinosa-Lily Thorn (S.)

- Clerodendron macrosiphon

Clerodendron ugandense (S.)

*Crotons (Codiaeum) - several races

17 Cyanophyllum magnificum

/1 *Duranta Ellisii

$15 *$ Duranta repens

Ehretia microphylla-per cutting

. 040

. 025

.. 025

. 040

.. 050

. 025

.. 025

.. 001

15- Eranthemum malaccense .

Ervatamia divaricata--Susun Kelapa ..

35- Euphorbia pulcherrima-Poinsettia

* Exeoccaria bicolor

13 Gardenia florida-Bunga China -

4 Gustavia gracillima

12 Gustavia speciosa

3- Hamelia patens (S.)

- Hibiscus mutabilis

4 *Hibiscus rosa-sinensis, various races, rooted cutting

- Hibiscus, standard plants ..

- *Hibiscus schizopetalus

- Hibiscus sp. (small pink flowers)

2s- Holmskioldia sanguinea

7 Honckenya ficifolia

10 Ipomoea carnea

2 Ixora chinensis

10 Ixora coccinea and varieties

10 Ixora congesta

Ixora finlaysoniana

4. Ixora javanica and varieties

Ixora nigricans

4 Jasminum bifarium

3- Jasnininum glandulosum

10 Jasminum pubescens

20 Jasminum sambac

6 Jatropha multifida (S.)

Jatropha pandurifolia and varieties

17 Kopsia fruticosa

9 Lagerstroemia indica (P.).

40 Lantana camara, varieties

10 Malpighia coccigera

10 * Malpighia nitida

5- Malvaviscus arborea (S.)

25. Malvaviscus Conzattii (S.)

\& Manihot utilissima variegata_-Variegated Tapioca.

Muehlenbeckia platyclada .

15 Murraya paniculata-Kemuning

M- Mussaenda erythrophylla. .

10 Mussaenda luteola

Nerium oleander-Oleander

$\$$ Ochna pumila (S.)

Panax Guilfoylei

Panax fruticosum

15- Petrea rugosa (P.)

10 Phaleria Blumei

15 Plumeria acutifolia-mrangipanni

27 Plumeria obtusifolia $2 . \cdots$

20. Plumeria rubra (Red Frangipanni) and varieties.

3 Randia macrantha-Angels Trumpets ..

*Ravenia spectabilis (S.)

15 Rondeletia odorata ...

4 Sambucus javanica-Javanese Elder .

15- Sambucus velutina

8. Solanum Wrighti-Potato Tree

Tecoma Smithii (P.)

30 Tecoma stans-Yellow Bells

* Tecomaria capensis

Thevetia peruviana-yellow, white and apricot .

20 Thunbergia affinis

25 cents to 050

75 cents to 100

. 025

025

.. 025

. 025

. 025

. 040

. 040

. 040

. 040

.. 040

. 040

. 025

- 025

. 025

. 025

.. 025

.. 025

.. 040

.. 020

.. 025

.. 025

.. 025

.. 025

.. $050^{\circ}$

.. 040

.. 050

.. 040 .

.. 025

.. 040

.. 040 .

.. 025

.. 025

.. 060

.. 060

. 025

$\therefore \quad 040$

$\therefore \quad 040$

. 025

.. 025

.. 025

.. 060

.. 025

.. $025=$

.. 025

.. 025

.. 025

.. 025

$\therefore \quad 025$

. 040 
SHRUBS.-continued

Thunbergia erecta

Thunbergia Kirkii

Thunbergia Vogeliana

Tithonia diversifolia

Turnera aurantiaca

Uroskinnera spectabilis

$\ldots$
$\cdots$
$\cdots$
$\cdots$
$\cdots$

\section{CLIMBERS}

\begin{tabular}{|c|c|c|c|c|}
\hline 20 & Afgekia sericea & & $\cdots$ & .. \\
\hline s. & Allamanda cathartica & . & . & . \\
\hline 15 & Antigonum leptopus-Honolulu Creepe & & .. & . \\
\hline 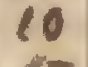 & Antigonum leptopus-var. album (whi & ite) & . & . \\
\hline 3 & Aristolochia grandiflora (S.) & . & . & \\
\hline 10 & Bauhinia Kockiana & . & . & . \\
\hline 10 & Beaumontia multiflora & . & . & . \\
\hline 12 & Bignonia magnifica-Purple Bignonia & -. & . & \\
\hline 12 & Bignonia tweediana (S.) $\ldots$ & -. & . & . \\
\hline $5^{-}$ & Bignonia unquis-cati & . & $\cdots$ & \\
\hline & Camoensia maxima & . & $\cdots$ & . \\
\hline 25 & Chonemorpha spp. & & . & . \\
\hline 25. & Clematis triloba-Indian Traveller's Jo & & $\cdots$ & . \\
\hline 10 & Clerodendron splendens & . & $\cdots$ & \\
\hline 6 & Clerodendron Thomsonae .. & . & $\cdots$ & $\cdots$ \\
\hline 4 & Clitorea ternatea & $\cdots$ & $\cdots$ & $\cdots$ \\
\hline 3 & Congea tomentosa & . & . & . \\
\hline 8 & Congea velutina & $\cdots$ & $\cdots$ & . \\
\hline & Cryptostegia madagascariensis (P.) & $\cdots$ & $\cdots$ & $\cdots$ \\
\hline$c$ & Faradaya splendida & & $\cdots$ & . \\
\hline & $\begin{array}{l}\text { Ficus pumila (1vy-like creeper) Creepi } \\
\text { Gloriosa superba }\end{array}$ & ing Fig & tubers & $\begin{array}{l}\cdots \\
\ldots\end{array}$ \\
\hline & Gloriosa virescens (S.) $\quad \ldots$ & . & tubers & . \\
\hline 4 & Hosea Lobbii (S.) & . & $\cdots$ & . \\
\hline 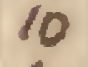 & Ipomoea digitata-Morning Glory (mal & auve) & $\cdots$ & . \\
\hline 4 & Ipomoea Horsfalliæ, Briggsii & . & . & . \\
\hline & Ipomoea Learii-Morning Glory (hlue) & & $\cdots$ & . \\
\hline 20 & Ipomoea pulchella $\quad$.. & $\cdots$ & . & . \\
\hline 10 & Jacquemontia violacea & . & ․ & . \\
\hline 4 & Jasminum rex ... & & . & $\cdots$ \\
\hline 15- & Lonicera japonica-Japanese Honeysuc & ckle & . & . \\
\hline 9 & Lonicera macrantha-Indian Honeysuch & kle & $\cdots$ & . \\
\hline 20 & Odontadenia speciosa $\quad$.. & . & $\cdots$ & . \\
\hline 15 & Pandorea jasminoides & . & . & . \\
\hline & Pandorea pandorana & . & . & . \\
\hline 10 & Pandorea ricasoliana (S.) $\because$ & $\cdots$ & . & . \\
\hline 5 & Passiflora laurifolia-Buah Susu & . & . & . \\
\hline $15^{\circ}$ & Passiflora quadrangularis-Grenadilla & & . & . \\
\hline 14 & Passiflora quadriglandulosa & . & . & . \\
\hline 10 & Petrea volubilis & . & . & . \\
\hline 20 & Porana volubilis-Bridal Creeper & . & . & - \\
\hline 10 & Quisqualis indica-Drunken Sailor & . & . & - \\
\hline 10 & Roupellia grata & . & $\cdots$ & $\cdots$ \\
\hline & Stephanotis floribunda (S.) & . & . & . \\
\hline 20 & Stigmaphyllon ciliatum & . & . & - \\
\hline 4 & Stigmaphyllon lancifolium & . & $\cdots$ & - \\
\hline 14 & Stigmaphyllon sagraeanum & . & . & . \\
\hline 7 & Thunbergia grandiflora & $\cdots$ & . & $\cdots$ \\
\hline 8 & Thunbergia grandiflora var. alba & $\cdots$ & $\cdots$ & - \\
\hline & Thunbergia laurifolia & $\cdots$ & $\cdots$ & - \\
\hline & Tristellateia australasica $\quad$. & . & . & . \\
\hline
\end{tabular}

PALMS

Acoelorraphe Wrightii (S.) $\quad$.. $\quad \ldots \quad \ldots 040$

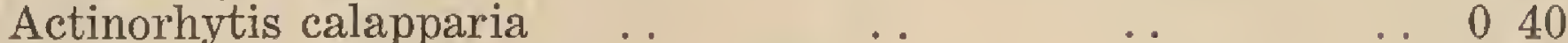

$\begin{array}{llllll}\text { Archontophoenix Alexandrae } & \ldots & \ldots & \ldots & 0 & 40\end{array}$

Arecá catechu-Betel-nut Palm $\quad . \quad \ldots \quad \ldots \quad$. 040

Arenga saccharifera-Kabong, Sugar Palm _. $\quad$.. 040

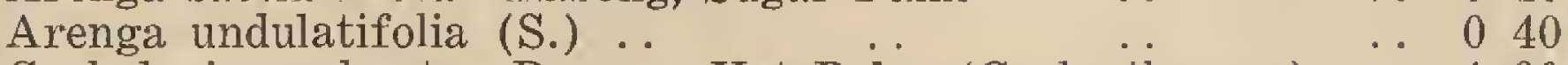

Carludovica palmata-Panama Hat Palm (Cyclanthaceae) . . 100

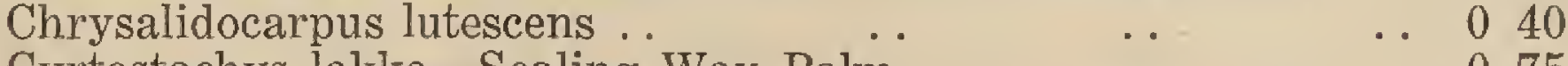

Cyrtostachys lakka-Sealing Wax Palm $\quad \ldots . \quad \ldots 075$

Elaeis guineensis-African Oil Palm (for ornamental purposes) 040 
PALMS.-continued

Heterospathe salomonensis (S.)

Hyophorbe Verschaffeltii (S.)

to Licuala grandis

7 Ticuala spinosa

4 Livistona chinensis

Livistona cochin-chinensis ..

Livistona rotundifolia

20 Livistona Woodfordii

14 Oncosperma tigillaria-Nibong

4 Oreodoxa oleracea-Cabbage Palm

- Oreodoxa regia-Royal Palm

12. Pinanga Kuhlii

s- Pinanga patula

2. Ptychosperma McArthuri

10 Ptychosperma sanderiana.

Rhapis flabelliformis-suckers, not in pots

7 Rhopaloblaste hexandra (S.)

1. Stevensonia grandifolia

8 Verschaffeltia splendida

$\$ c$.

. 040

. 075

. 040

. 040

- 040

0 40

. 040

- 040

. 040

. 040

. 040

- 040 .

. 040

$\therefore \quad 040$

. $\quad 040$

.. 020

. 040

. 075

075

\section{HERBACEOUS PLANTS AND DWARF SHRUBS}

Ageratum, dwarf (P.)

Alternanthera paronichioides

- Angelonia salicariaefolia

Artemisia lactiflora

Aster bellidioides

- Aster sp. (dwarf)

Aster, perennial-Michaelmas Daisy

Asystasia intrusa

Barleria cristata, four varieties

Barleria lupulina

- Beloperone guttata

- Cestrum nocturnum

Clerodendron paniculatum-Pagoda Flower

- Coleus Blumei, various races

- Coreopsis grandiflora (perennial)

- Crinum asiaticum-Crinum Lily

- Crinum giganteum-Java Lily

- Crossandra nilotica (P.)

- Crossandra undulifolia-Singàpore Geranium

- Crossandra undulifolia-yellow variety

Cuphea platycentra (P.)

- Cuphea sp.

Daedalacanthus nervosus .

Eranthemum atropurpureum

Eranthemum hypocrateriforme

Eranthemum reticulatum

- Eranthemum Wattii

"Eucharis grandiflora-Amazon

" Galphimia glauca

"Hedychium coronarium

" Helianthus angustifolius-Perennial Sunflower

" Heliconia psittacorum-"Japanese Canna"

". Hippeastrum

"Hymenocallis littoralis-Spider Lily ..

- Lantana sellowiana

- Mirabilis jalapa-Marvel of Peru

- Orthosiphon stamineus-Cat's Whiskers

" Oxalis dispar (S.)

- Pancratium zeylanicum

- Pennisetum macrostachyum (S.)

Pentas alba

- Pentas carnea

- Pentas coccinea.

" Platycodon grandiflorum

- Plumbago capensis-Blue Plumbago

- Plumbago rosea-Pink Plumbago

- Rudbeckia Newmanii-Black-Eyed Susan

4 Ruellia macrophylla

" Ruellia malacosperma

$\cdots$

$\cdots$
025

12 for 010

suckers .. 0005

. 025

$0 \quad 05$

12 for 010

suckers .. 005

.. 015

.. 020

.. 025

- 020

5 cents or 010

040

. 025

.. 001

.. 010

. 040

. 040

.. 025

. 025

. 040

. 025

. 015

.. 025

$\therefore \quad 025$

.. 025

.. 025

. 025

. 040

.. 025

. 020

per root $\cdots \quad 005$

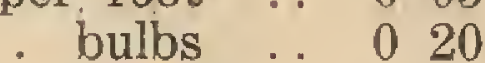

bulbs .. 0010

. 020

.. 020

. 025

.. 025

bulbs .. 0010

per stool .. 005

.. 020

. $\quad \ldots 020$

. $\quad \ldots 025$

. 025

. . 050

.. $\quad .0025$

$\cdots 0$

. $\quad \because 025$

$\cdots 025$ 
HERBACEOUS PLANTS AND DWARF SHRUBS.-continued

Ruellia tuberosa

Russellia juncea

Russellia sarmentosa

Sanchezia nobilis

Turnera ulmifolia

4 Turnera trioniflora

Tussacia pulchella

Verbena tenera.

Vinca rosea-Periwinkle

Wedelia biflora, double

Zephyranthes, white, yellow and pink

- Zephyranthes, hybrids and large flowered forms

$\cdots$
$\cdots$
$\cdots$
$\cdots$
$\cdots$
$\cdots$
per bulb

. 005

. 025

. 020

. 040

. 020

.. 020

.. 025

. 005

. 020

.. 025

. $\quad 001$

. 005

\section{SEEDS OF HERBACEOUS PLANTS}

\begin{tabular}{|c|c|c|c|}
\hline Browallia elata & & per packet & 0 \\
\hline Celosia & . & " & .. \\
\hline Coreopsis tinctoria & . & ," & . \\
\hline Cosmos sulphureus & & , & . \\
\hline Gynandropsis speciosissima & (cat's whisker's) & ", & . \\
\hline Hymenatherum tenuilobum & $\cdots$ & " & . \\
\hline mpatiens balsamina (doubl & e Balsam) & ", & . \\
\hline Melampodium divaricatum & . & ", & .. \\
\hline Petunia $\quad \because$ & . & " & . \\
\hline Torenia Fournieri & . & " & . \\
\hline Zinnia linearis & .. & ", & $\ldots$ \\
\hline
\end{tabular}

\section{WATER GARDEN PLANTS}

Acorus calamus--Sweet Flag (S.) . . .

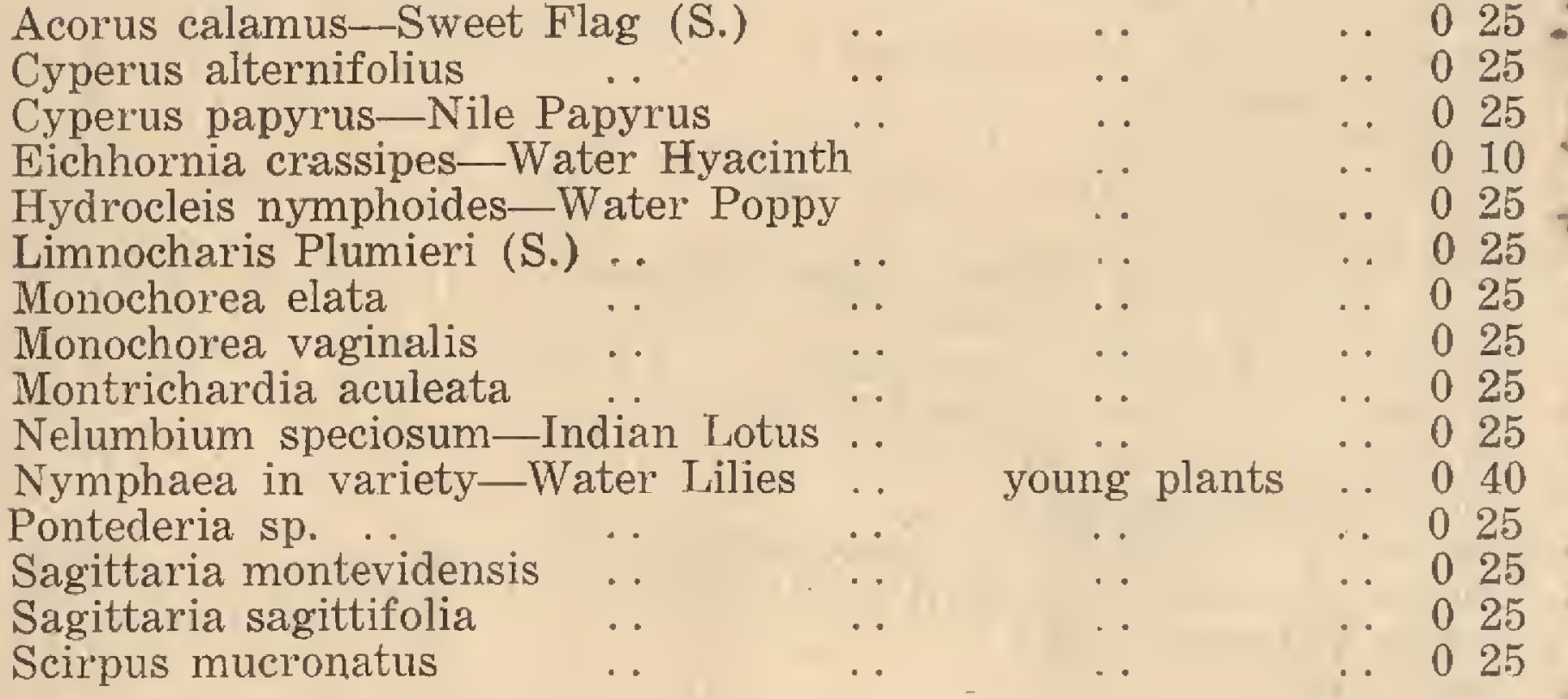

\section{ORCHIDS}

Arachnis flos-aeris-Scorpion Orchid Arachnis hookeriana (S.) - White Scorpion Orchid cuttings Arachnis Maingayi-Scorpion Orchid

Vanda hookeriana

Vanda hybrid Miss Joaquim

Vanda teres

Vanda teres var. Andersonii

$\begin{array}{cccc}\text { cuttings } & \ldots & 0 & 20 \\ \text { per cutting } & \ldots & 0 & 20 \\ , " & \ldots & 0 & 50 \\ , & & 0 & 05 \\ , & & 0 & 50 \\ \text {, } & & 0 & 50\end{array}$

Occasionally plants of other orchids are for: sale; prices on application.

[No. $768 / 38]$. 
The following is published for general information:-

\section{LIST OF PLANTS}

which may usually be obtained from the Botanic Gardens at Singapore and Penang.

\footnotetext{
(S.) and (P.) mean at Singapore or at Penang only.

* Means suitable for hedge-making.

$\doteqdot$ Unrooted cuttings for hedge-making, 100 for $75 \mathrm{cts}$.
}

Unless stated otherwise, the prices are for small plants in pots and the price may be altered according to the size of the plant. Rooted cuttings dug from the nursery and not in pots are charged at 20 to 40 cents, according to size. The cost of packing and all subsequent charges are extra.

Plants not included in the following lists are sometimes available.

Brownea grandiceps (S.)

- Cassia fistula-Indian Laburnum

2 - Cassia grandis-Pink Cassia

- Cassia javanica.

- Cassia nodosa-Pink Cassia

* Cassia renigera-Burmese Cassia

Cassia multijuga

* Cassia siamea-Johar

- Cassia spectabilis

$\ldots$
$\cdots$
$\cdots$
$\cdots$
rnum
..
Cassia
$\ldots$
..

Citharexylum quadrangulare

. Cochlospermum religiosum-Buttercup Tree

so Cratoxylon formosum

Cratoxylon ligustrinum

to Delonix regia-Flame of the Forest ..

(0) Enterolobium saman-Rain Tree

14 Erythrina indica-Coral Tree (Dadap) (P.)

4 Jacaranda filicifolia

- Lagerstroemia floribunda ..

is Lagerstroemia flos-reginae-Rose of India

- Lagerstroemia Loudonii

- Mesua ferrea-Ceylon Ironwood

- Peltophorum pterocarpum-Yellow Flame

Pterocarpus indicus-Angsana

Saraca declinata (S.)

6- Saraca indica (S.)

Saraca taipingensis (S.) …

Wormia suffruticosa

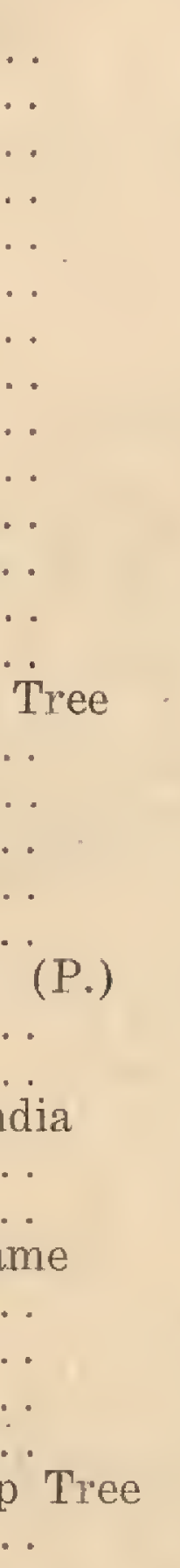

$\$ c$.

050

025

. 025

. 100

. 060

025

. 040

. 050

050

100

025

025

025

$0 \quad 25$

040

$\begin{array}{lll}0 & 40\end{array}$

$0 \quad 40$

$\therefore \quad 025$

. 040

. 025

. $\quad 040$

025

040

025

- 100

- 060

. 025

. 040

040

$\therefore \quad 100$

050

025

025

\section{FOLIAGE TREES}

Acacia auriculaeformis

Adenanthera pavonina--Saga Tree ..

Albizzia falcata-Batai .. .

Andira inermis (S.)

- Andira surinamensis (S.) ..

Arfeuillea arborescens

2. Calophyllum inophyllum-Penaga Laut

4. Canangium odoratum-Kenanga

*Casuarina equisetifolia-Common Ru .

Casuarina rumphiana (S.)

$30 *$ Casuarina sumatrana.

25" Cinnamomum iners - Wild Cinnamon

28 Dacrydium elatum-Ru Bukit

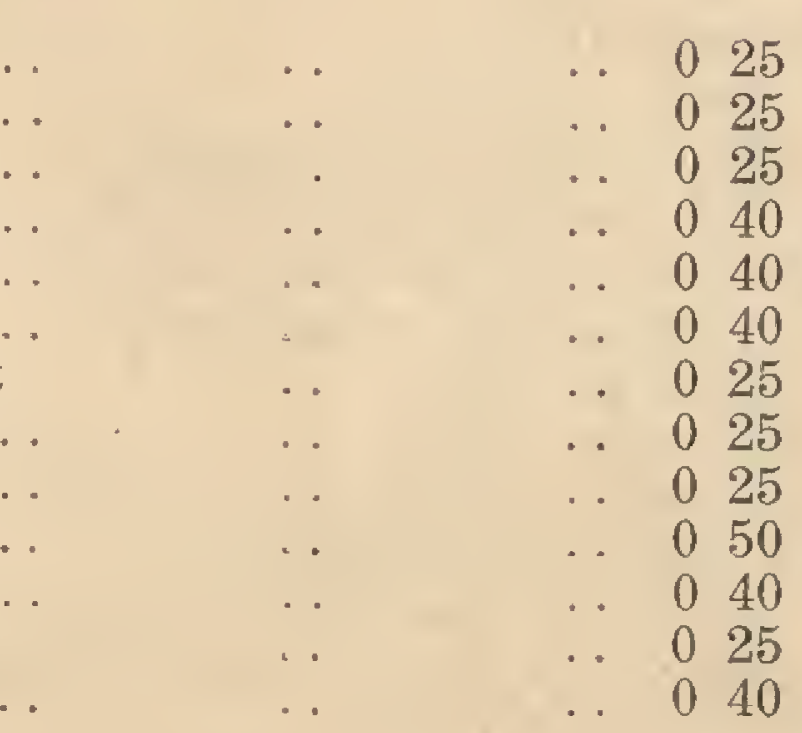


14 Elaeocarpus ganitrus

Eugenia densiflora ( $P$.

.. $\quad$.

Eugenia grandis-Jambu Laut

Eugenia Helferi (P.)

.

025

Eugenia longiflora-Kelat . .

25. Fagraea fragrans-Tembusu

Ficus irregularis (S.)

Filicium decipiens (P.)

35- Gliricidia sepium-Mexican Lilac

Gnetum Gnemon-Meninjau (P.)

14 Hymenaea courbaril (S.)

Melia excelsa (P.)

20 Millettia atropurpurea

4- Muntingia calabura-Cherry Tree

0.40

$0 " 25$

$\begin{array}{lll}0 & 25\end{array}$

100

$\begin{array}{lll}\cdots & 1 & 00 \\ . & 0 & 40\end{array}$

. 025

. 040

. 025

$0 \quad 25$

025

025

040

Parkia speciosa (P.)-Petai

$\begin{array}{ll}0 & 40 \\ 0 & 25\end{array}$

0) 60

060

100

025

025

$\begin{array}{lll}0 & 40\end{array}$

$\begin{array}{lll}0 & 01\end{array}$

040

040

040

\section{SHRUBS}

†*Acalypha siamensis (P.) from nursery bed

*Acalypha, various species-Cat's Tails .

$\checkmark$ Allamanda cathartica

4 *Allamanda Schottii

4 Allamanda violacea

*Allamanda Williamsii

Bauhinia acuminata (white)

Bauhinia tomentosa

*Bixa orellana-Anatto (Kesumba) from nursery bed

30 Bougainvillea Calcutta No. 2 (S.)

18 Bougainvillea Calcutta No. 13 (S.) .

68 Bougainvillea Cypheri (S.)

20 Bougainvillea formosa

is Bougainvillea glabra, pale rose variety

Bougainvillea glabra, var. magnifica

\&4*Bougainvillea glabra, var. Sanderiana

6 Bougainvillea lateritia

22 *Bougainvillea Mrs. Butt ..

10 Bougainvillea Mrs. Fraser ..

4 Bougainvillea Mrs. Lancaster

22 *Bougainvillea Mrs. McLean ..

1s * Bougainvillea Rosa Catalina

8 Bougainvillea Thomasii

45- Brunfelsia americana

7 Brunfelsia eximia (blue)

4s- Brunfelsia undulata (white, large flowers)

4 Caesalpinia pulcherrima-Peacock Flower

7 Caesalpinia pulcherrima var. lutea

Calliandra haematocephala (P.)

Cassia auriculata

12. Cassia bicapsularis

Cassia biflora

20 Cassia fruticosa

8 Cassia splendida

6 Clerodendron macrosiphon

3 Clerodendron ugandense (S.)

*Crotons (Codiaeum) - several races

is- Cyanophyllum magnificum

6 * Duranta Ellisii

10 * Duranta repens

$\uparrow$ Ehretia microphylla_-per cutting

$$
\cdots
$$


SHRUBS.-continued

- Gardenia florida-Bunga China

. $\quad 025$

2 Gustavia gracillima

14 Gustavia speciosa

Hamelia patens (S.)

Hibiscus mutabilis

. $\quad \cdots$

$4+*$ Hibiscus rosa-sinensis, various races, rooted cuttings

- Hibiscus, standard plants ..

Hibiscus sp. (small pink flowers)

025

15 - Holmskioldia sanguinea .. .

+ Honckenya ficifolia ..

Ipomoea carnea

Ixora chinensis

15- Ixora coccinea and varieties

Ixora congesta

14 Ixora javanica and varieties

- Jasminum bifarium

14 Jasminum glandulosum ..

6. Jasminum pubescens

14. Jasminum sambac

6 Jatropha multifida

5- Jatropha pandurifolia and varieties

12 Kopsia fruticosa

Lagerstroemia indica (P.) . .

25- Lantana camara, varieties

12. Malpighia coccigera

4. * Malpighia nitida

2. Malvaviscus arborea (S.) .

(6) Malvaviscus Conzattii

3 Manihot utilissima variegata-Variegated Tapioca .

12. Murraya paniculata-Kemuning

60 Mussaenda erythrophylla

Mussaenda luteola

4 Nerium oleander-Oleander

44. Ochna pumila (S.)

Panax Guilfoylei

Panax fruticosum

7 Petrea rugosa (P.)

20. Phaleria Blumei

Plumeria acutifolia_-Frangipanni _. .

20 Plumeria obtusifolia

12 Plumeria rubra (Red Frangipanni) and varieties .

6 Quassia amara-Bitter Quassia

Randia macrantha-Angels Trumpets .

2 * Ravenia spectabilis (S.)

12 Rondeletia odorata

Sambucus javanica-Javanese Elder .

to Sambucus velutina

Solanum Wrighti i-Potato Tre

- Tecoma Smithii (P.)

(2) Tecoma stans-Yellow Bells

*Tecomaria capensis

10 Thevetia peruviana-yellow, white and apricot

10 Thunbergia affinis

12. Thunbergia erecta

Thunbergia Kirkii

Tithonia diversifolia

Turnera aurantiaca

Uroskinnera spectabilis

\section{CLIMBERS}

Afgekia sericea

Allamanda cathartica var. Hendersoni .

Antigonum leptopus-Honolulu Creeper

Antigonum leptopus-var. album (white)

Aristolochia grandiflora (S.)

Bauhinia Kockiana

Beaumontia multiflora

Bignonia magnifica-Purple Bignonia ..

Camoensia maxima

Chonemorpha macrophylla

Chonemorpha penangensis

Clematis triloba-Indian Traveller's Joy

Clerodendron splendens

Clerodendron Thomsonae

Clitorea ternatea

Congea tomentosa

Congea velutina

Cryptostegia madagascariensis (P.) . 
Faradaya splendida

\section{.}

040

Ficus pumila (ivy-like creeper) Creeping Fig - .

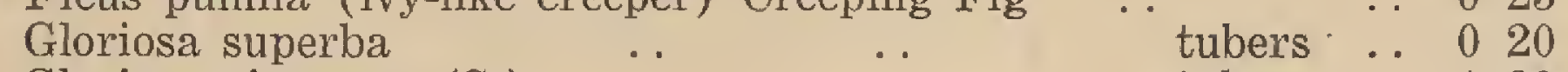

Gloriosa virescens

10 Hosea Lobbii (S.)

12 Ipomoea digitata-Morning Ġlory (mauve)

20 Ipomoea Horsfalliæ, Briggsii

4 Ipomoea Learii-Morning Glory (blue)

Ipomoea pulchella, from nursery beds .

Jacquemontia violacea

8 Jasminum rex

6 Lonicera japonica-Japanese Honeysuckle

6 Lonicera macrantha-Indian Honeysuckle

- Odontadenia speciosa

10 Pandorea jasminoides

- Pandorea pandorana

15- Pandorea ricasoliana (S.)

is- Passiflora laurifolia_Buah Susu

20 Passiflora quadrangularis-Grenadilla

Passiflora quadriglandulosa

Petrea volubilis

- Porana volubilis-Bridal Creeper

Quisqualis indica-Drunken Sailor

15- Roupellia grata

Stephanotis floribunda

$r$ Stigmaphyllon ciliatum

- Stigmaphyllon lancifolium

Stigmaphyllon sagraeanum

10 Thunbergia grandiflora

10 Thunbergia grandiflora var. alba

Thunbergia laurifolia

Tristellateia australasica

tubers

- 100

060

025

. 040

025

005

. 025

.. 040

. 025

025

100

040

040

$\begin{array}{lll}0 & 40\end{array}$

025

040

025

025

025

025

040

100

025

025

0 25

025

025

025

025

\section{PALMS}

Acoelorraphe Wrightii (S.)

$\begin{array}{lll}0 & 40\end{array}$

Actinorhytis calapparia

40

040

Areca catechu-Betel-nut Palm

Arenga saccharifera-Kabong, Sugar Palm

Arenga undulatifolia (S.) . .

Carludovica palmata-Panama Hat Palm (Cyclanthaceae)

Chrysalidocarpus lutescens.

20 Cyrtostachys lakka-Sealing Wax Palm

Elaeis guineensis-African Oil Palm (for ornamental purposes) Heterospathe salomonensis (S.)

us- Licuala grandis

Licuala spinosa

Livistona chinensis

Livistona cochin-chinensis .

10 Livistona rotundifolia

20 Livistona Woodfordii

Oncosperma tigillaria-Nibong

Oreodoxa oleracea-Cabbage PaIn

Oreodoxa regia_-Royal Palm

10 Pinanga Kuhlii

2 Pinanga patula

20 Ptychosperma McArthuri

20 Ptychosperma sanderiana

Rhapis flabelliformis-suckers, not in pots

10 Rhopaloblaste hexandra (S.)

10 Stevensonia grandifolia

Verschaffeltia splendida

\section{HERBACEOUS PLANTS AND DWARF SHRUBS}

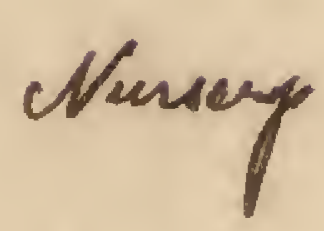

Ageratum, dwarf

Alternanthera paronichioides

Angelonia salicariaefolia

Artemisia lactiflora

- Aster bellidioides

- Aster sp. (dwarf)

- Aster, perennial-Michaelmas Daisy

- Asystasia intrusa

- Barleria cristata, four varieties

Barleria lupulina

- Begonia-bedding variety ..

- Beloperone guttata

Canna, hybrids, various races, roots

is Cestrum nocturnum

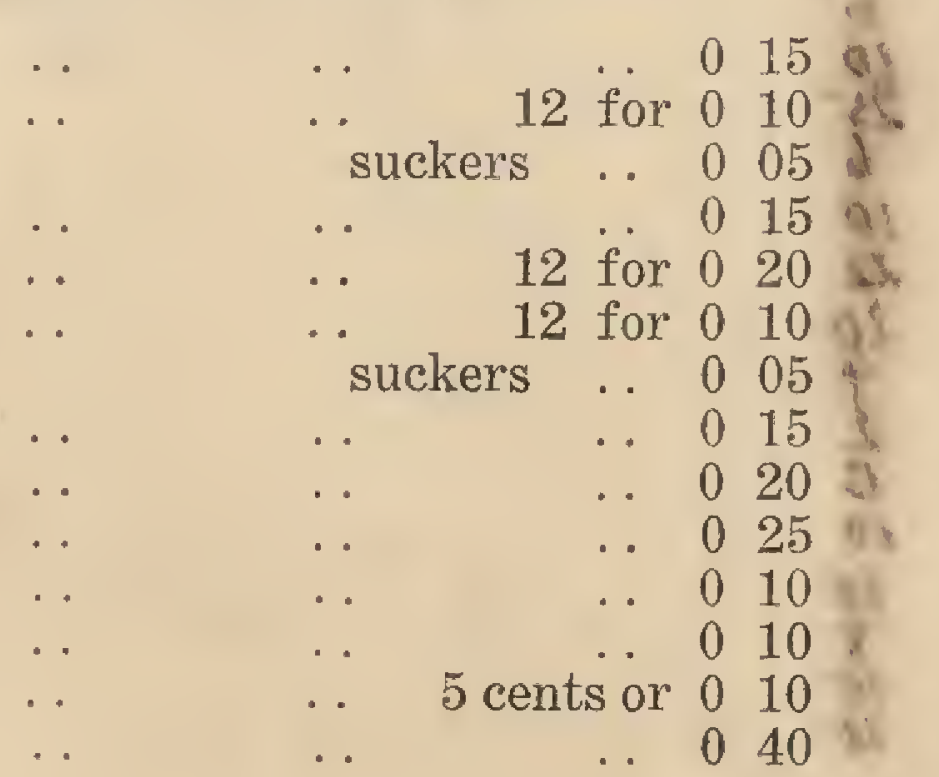


HERBACEOUS PLANTS AND DWARF SHRUBS.-continued

Clerodendron paniculatum-Pagoda Flower

Coleus Blumei, various races

Coreopsis grandiflora (perennial)

Crinum asiaticum-Crinum Lily

4 Crinum giganteum-Java Lily

Crossandra nilotica (P.)

Crossandra undulifolia-Singapore Geranium

Crossandra undulifolia-yellow variety

Cuphea platycentra

- Cuphea sp. (red)

- Daedalacanthus nervosus

- Eranthemum atropurpureum

- Eranthemum hypocrateriforme

Eranthemum reticulatum

12 Eranthemum Wattii

Eucharis grandiflora-Amazon Lily .

Galphimia glauca

- Hedychium coronarium, suckers

4. Helianthus angustifolius-Perennial Sunflower

Heliconia psittacorum-“Japanese Canna"

- Hippeastrum

- Hymenocallis littoralis-Spider Lily .

Lantana sellowiana

- Mirabilis jalapa-Marvel of Peru

1. Oxthosiphon stamineus-Cat's Whiskers

Pancratium zeylanicum

- Pennisetum macrostachyum (S.)

Pentas alba

Pentas carnea.

Pentas coccinea.

Platycodon grandiflorum

Plumbago capensis-Blue Plumbago

Plumbago rosea-Pink Plumbago

Rudbeckia Newmanii-Black-Eyed Susan

- Ruellia macrophylla

- Ruellia malacosperma

Russellia juncea

Russellia hybrid

Russellia sarmentosa

Sanchezia nobilis

- Turnera ulmifolia

Turnera trioniflora

Tussacia pulchella

- Verbena tenera

Vinca rosea-Periwinkle

* Wedelia biffora, double

- Zephyranthes, white, yellow and pink

4 Zephyranthes, hybrids and large flowered forms

UUS.-continued

\section{SEEDS OF HERBACEOUS PLANTS}

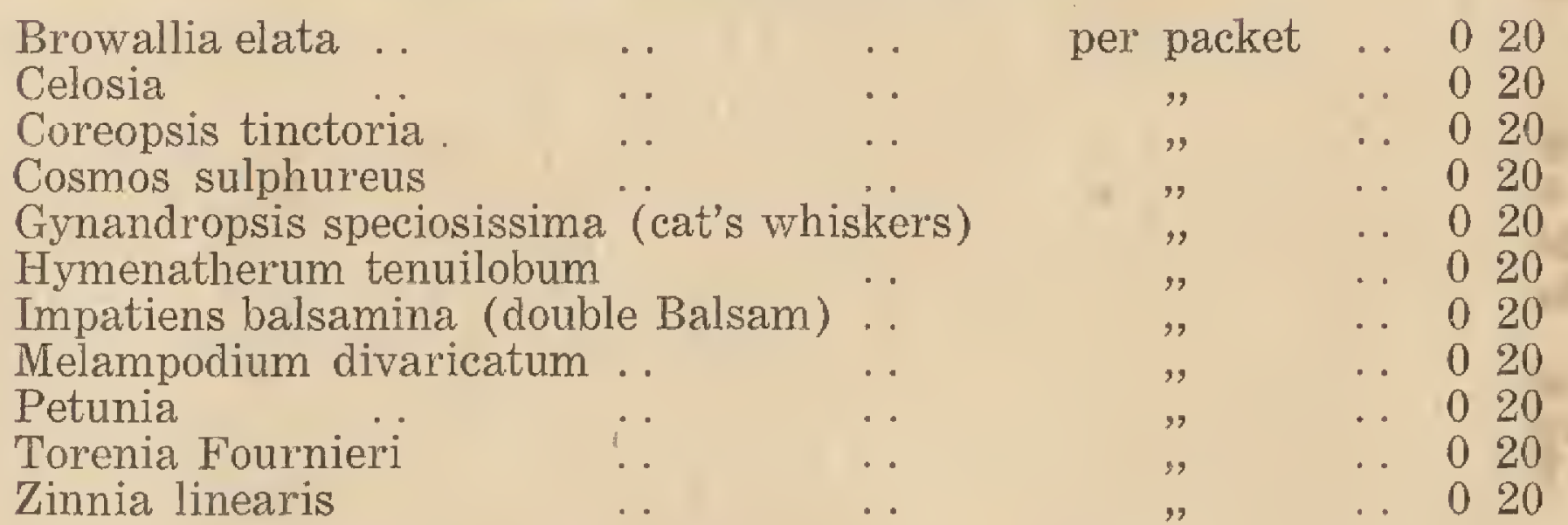

\section{WATER GARDEN PLANTS}

Acorus calamus-Sweet Flag (S.) ". $\quad$. . $\quad \ldots .025$

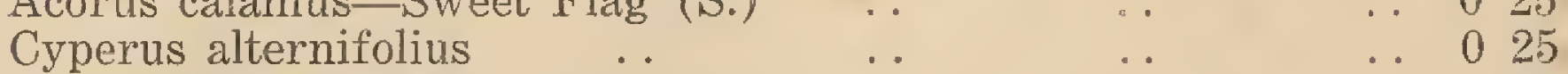

Cyperus papyrus-Nile Papyrus $\quad \ldots \quad \ldots 050$

Eichhornia crassipes-Water Hyacinth $\quad \ldots \quad \ldots 010$

Hydrocleis nymphoides-Water Poppy $\ldots \ldots 025$

Limnocharis Plumieri (S.) . . . . . 025

$\begin{array}{lllllll}\text { Monochorea elata } & \ldots & \ldots & \ldots & & \ldots & 25\end{array}$

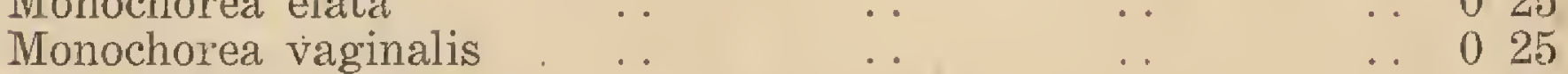

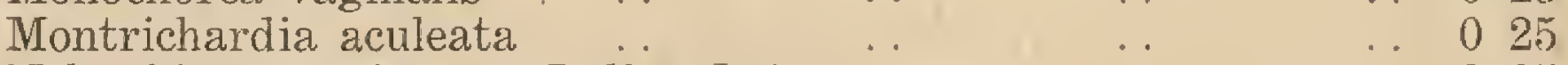

Nelumbium speciosum-Indian Lotus .. $\quad \ldots .0 \quad \ldots 025$

Nymphaea in variety-Water Lilies .. $\quad$ young plants .. 040

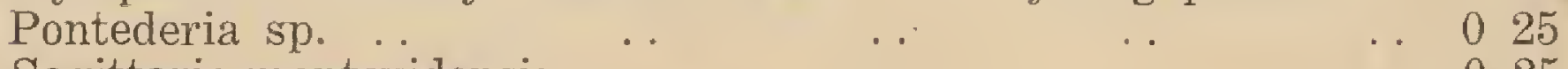

$\begin{array}{lllllll}\text { Sagittaria montevidensis } & \ldots & \ldots & \ldots & \ldots & 25 \\ \text { Sagittaria sagittifolia } & \ldots & \ldots & \ldots & \ldots & 0 & 25 \\ & & \ldots & \ldots & \ldots & 0 & 25\end{array}$ 


\section{ORCHIDS}

Arachnis flos-aeris-Scorpion Orchid

$\$ c$.

Arachnis Hookeriana (S.) - White Scorpion Orchid

Arachnis Maingayi-Scorpion Orchid

Spathoglottis plicata

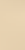

Spathoglottis plicata white . .

Vanda Hookeriana

Vanda hybrid Miss Joaquim

Vanda hybrid Josephine

Vanda teres

Vanda teres var. Andersonii

(2)

.

. 020

. 040

$\cdot$

060

per cutting .. 0050

" . $\quad 005$

. 050

Occasionally plants of other orchios are for sale; prices on application.

[No. C.S.O. $768 / 38]$. 
The following is published for general information:-

\section{LIST OF PLANTS}

which may usually be obtained from the Botanic Gardens at Singapore and Penang.

\footnotetext{
(S.) and (P.) mean at Singapore or at Penang only.

* Means suitable for hedge-making.

† Unrooted cuttings for hedge-making, 100 for $75 \mathrm{cts}$.
}

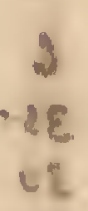

Unless stated otherwise, the prices are for small plants in pots and the price may be altered according to the size of the plant. Rooted cuttings dug from the nursery and not in pots are charged at 20 to 40 cents, according to size. The cost of packing and all subsequent charges are extra.

Plants not included in the following lists are sometimes available.

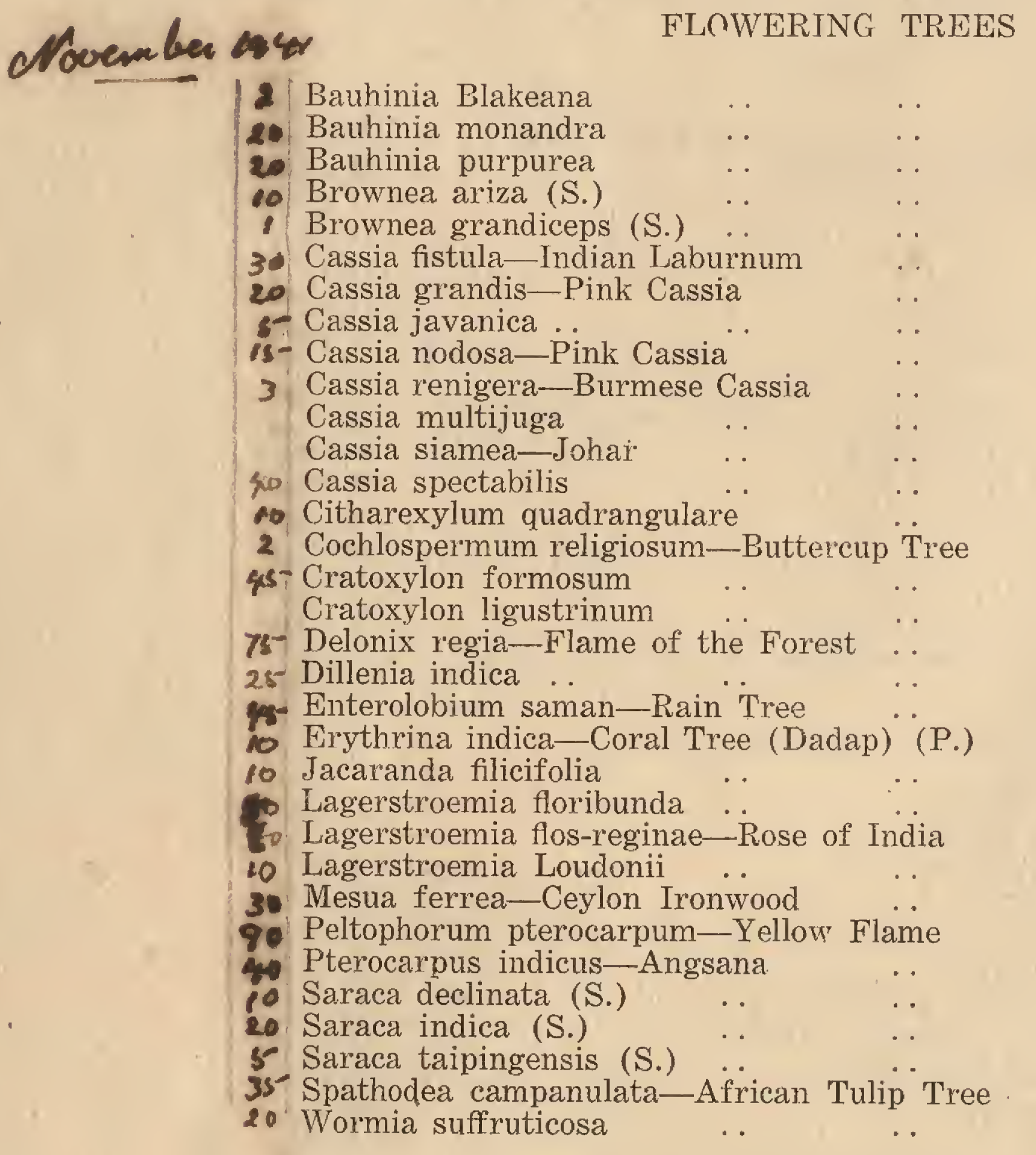

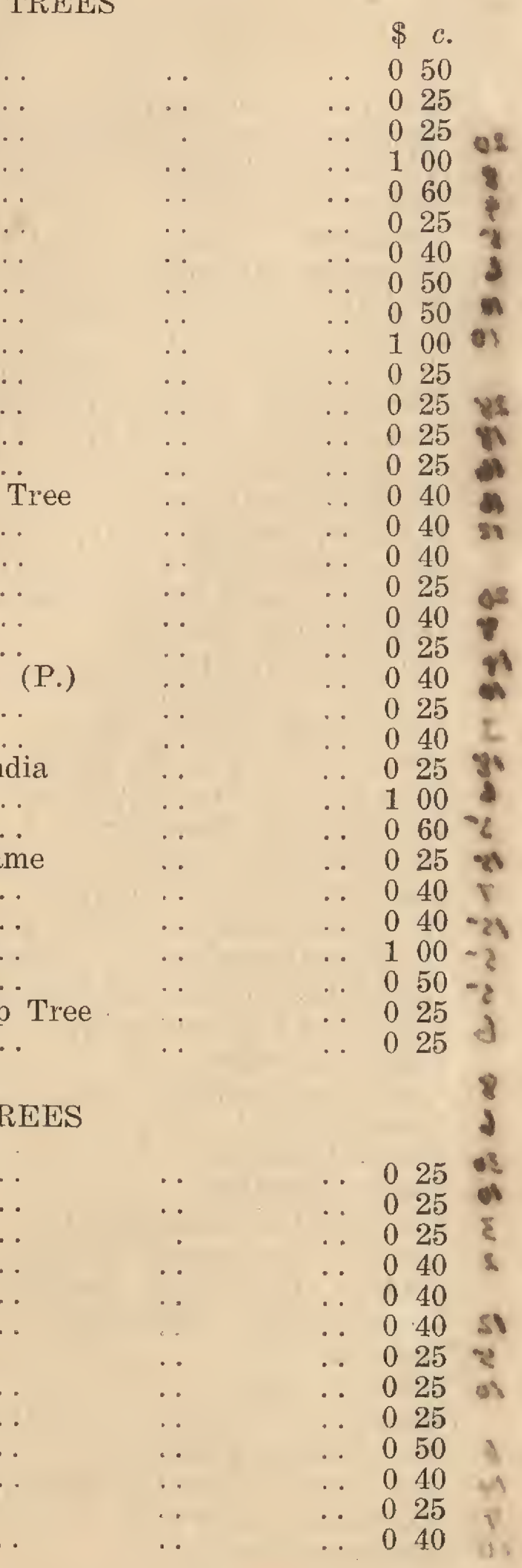

Acacia auriculaeformis

Adenanthera pavonina-Saga Tree

Albizzia falcata-Batai

12 Andira inermis (S.)

20. Andira surinamensis (S.) .

35- Arfeuillea arborescens

Calophyllum inophyllum Penaga Laut

6. Canangium odoratum-Kenanga

*Casuarina equisetifolia-Common $\mathrm{Ru}$. .

Casuarina rumphiana (S.)

30 *Casuarina sumatrana

eo Cinnamomum iners-Wild Cinnamon

28. Dacrydium elatum-Ru Bukit

\section{TREES}


FOLIAGE TREES.-continued

$\$ c$.

(1) Elaeocarpus ganitrus

Eugenia densiflora (P.)

25

Eugenia grandis-Jambu Lau

Eugenia Helferi (P.)

Eugenia longiflora-Kelat . .

20 Fagraea fragrans-Tembusu

6. Ficus irregularis (S.)

Filicium decipiens (P.)

30 Gliricidia sepium-Mexican Lilac

Gnetum Gnemon--Meninjau (P.)

12 Hymenaea courbaril (S.)

Melia excelsa (P.)

(v) Millettia atropurpurea

Muntingia calabura-Cherry Tree

. . .

025

.. 040

. 025

. 040

. 025

. 025

. 100

. 040

. 025

.. 040

. 025

. 025

025

025

Parkia speciosa (P.)-Petai

6 *Pithecellobium dulce-Madras Thorn.

33-* Podocarpus gracilior

$30 *$ Podocarpus polystachyus

18 Podocarpus Rumphii

Polyalthia longifolia (P.) . .

040

.025

060

060

100

025

025

040

$\begin{array}{lll}0 & 01\end{array}$

$\begin{array}{lll}0 & 40\end{array}$

040

*Streblus asper-per cutting

Swietenia macrophylla-Broad leaved Mahogany ..

10 Tamarindus indica-Tamarind

Terminalia catappa-Bengal Almond

\section{SHRUBS}

†*Acalypha siamensis (P.)

from nursery bed

() 40

Acalypha, various species-Cat's Tails .

Allamanda cathartica

*Allamanda Schottii

Allamanda violacea

* Allamanda Williamsii

Bauhinia acuminata (white)

10 Bauhinia tomentosa

*Bixa orellana-Anatto (Kesumba)

28 Bougainvillea Calcutta No. 2 (S.)

* Bougainvillea Calcutta No. 13 (S.)

4 Bougainvillea Cypheri (S.)

* Bougainvillea formosa

* Bougainvillea glabra, pale rose variety

Bougainvillea glabra, var. magnifica

at: Bougainvillea glabra, var. Sanderiana

* Bougainvillea lateritia

(v *Bougainvillea Mrs. Butt

Bougainvillea Mrs. Fraser .

3 Bougainvillea Mrs. Lancaster

- * Bougainvillea Mrs. McLean ..

+ Bougainvillea Rosa Catalina

s- Bougainvillea Thomasii

* Brunfelsia americana

7 Brunfelsia eximia (blue)

15* Brunfelsia undulata (white, large flowers)

5- Caesalpinia pulcherrima-Peacoch Flower

s- Caesalpinia pulcherrima var. lutea

6 Calliandra haematocephala (P.)

Cassia auriculata

8 Cassia bicapsularis

6 Cassia biflora

3. Cassia fruticosa

- Cassia splendida

3 Clerodendron macrosiphon

Clerodendron ugandense (S.)

Crotons (Codiaeum) — several races

12 Cyanophyllum magnificum

5 *Duranta Ellisii

- *Duranta repens

Ehretia microphylla-per cutting

$\begin{array}{llll}\cdots & 025 & 25\end{array}$

025 o

025

040

025 as

025

025

$\begin{array}{lll}0 & 05\end{array}$

from nursery bed

050

050

$050 \mathrm{~m}$

0.75 s

075 ㅇ

100

025

050

025 म

075

100

025

025

050 व

. 040

040

050

.. 020

- 020 है

$\therefore \quad 040$

025

. 025

. 025

. 025

040

. 025

. 025

.. 040 as

.. $050-28$

. 025

. 025

.. 001

.. 025

6 Eranthemum malaccense

4 Ervatamia divaricata-Susun Kelapa.

9 Euphorbia pulcherrima-Poinsettia

10 *Excoecaria bicolor

.

.

.. 025

.. 025 
- Gardenia florida-Bunga China

2 Gustavia gracillima

10 Gustavia speciosa

Hamelia patens (S.)

$\ldots$
$\cdots$
$\cdots$

Hibiscus mutabilis

025

Hibiscus rosa-sinensis, various races, rooted cuttings

c Hibiscus, standard plants.

* Hibiscus schizopetalus

- Hibiscus sp. (small pink flowers)

2. Holmskioldia sanguinea

(. Honckenya ficifolia

Ipomoea carnea

5 Ixora chinensis

Ixora coccinea and varieties

Ixora congesta

- Ixora javanica and varieties

c. Jasminum bifarium

i4 Jasminum glandulosum

10 Jasminum pubescens

14. Jasminum sambac

5- Jatropha multifida

3 Jatropha pandurifolia and varieties

10 Kopsia fruticosa

5 Lagerstroemia indica (P.) .

2. Lantana camara, varieties

12 Malpighia coccigera

10*Malpighia nitida

2o Malvaviscus arborea (S.) .

to Malvaviscus Conzattii

3 Manihot utilissima variegata-Variegated Tapioca .

- Murraya paniculata-Kemuning

- Mussaenda erythrophylla

10 Mussaenda luteola

4 Nerium oleander-Oleander

i4 Ochna pumila (S.)

Panax Guilfoylei

Panax fruticosum

6 Petrea rugosa (P.)

3. Phaleria Blumei

- Plumeria acutifolia_-Frangipanni

a Plumeria obtusifolia $\because$.

10 Plumeria rubra (Red Frangipanni) and varieties .

- Quassia amara-Bitter Quassia

6 Randia macrantha-Angels Trumpets ..

20*Ravenia spectabilis (S.)

Rondeletia odorata

Sambucus javanica-Javanese Elder

o Sambucus velutina

* Solanum Wrighti Potato Tree

Tecoma Smithii (P.)

Tecoma stans-Yellow Bells

12 *Tecomaria capensis

12 Thevetia peruviana-yellow, white and apricot

en Thunbergia affinis

co Thunbergia erecta

\& Thunbergia Kirkii

Tithonia diversifolia

Turnera aurantiaca

- Uroskinnera spectabilis

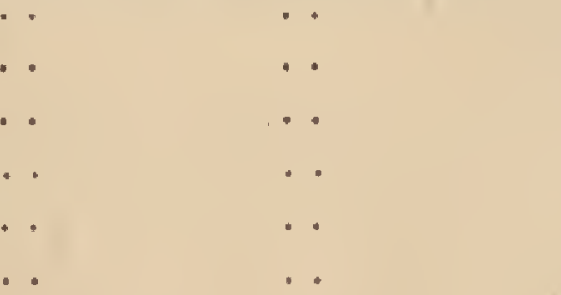

\section{CLIMBERS}

y. Afgekia sericea

Allamanda cathartica var. Hendersoni .

Antigonum leptopus-Honolulu Creeper

Antigonum Ieptopus-var. album (white)

6 Aristolochia grandiflora (S.)

2s- Bauhinia Kockiana

B Beaumontia multiflora

Bignonia magnifica-Purple Bignonia .

Camoensia maxima

8. Chonemorpha macrophylla

3 Chonemorpha penangensis

2 Clematis triloba-Indian Traveiler's Joy

Clerodendron splendens

Clerodendron Thomsonae

Clitorea ternatea

$\checkmark$. Congea tomentosa

8 Congea velutina

1o Cryptostegia madagascariensis (P.) ..

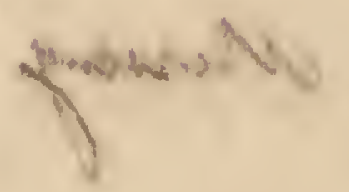


Faradaya splendida

Ficus pumila (ivy-like creeper) Creeping Fig Gloriosa superba

Gloriosa virescens (S.)
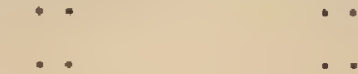

Hosea Lobbii (S.)

12 Ipomoea digitata-Morning Glory (mauve)

- Ipomoea Horsfalliæ, Briggsii

2 Ipomoea Learii-Morning Glory (blue)

Ipomoea pulchella, from nursery beds.

Jacquemontia violacea

r Jasminum rex

Lonicera japonica-Japanese Honeysuckle

Lonicera macrantha-Indian Honeysuckle

16 Odontadenia speciosa

1. Pandorea jasminoides

Pandorea pandorana

Pandorea ricasoliana (S.)

Passiflora laurifolia-Buah Susu

20 Passiffora quadrangularis-Grenadilla

४ Passiflora quadriglandulosa

3 Petrea volubilis

3. Porana volubilis-Bridal Creeper

6uisqualis indica-Drunken Sailor

is - Roupellia grata

4 Stephanotis floribunda

12 Stigmaphyllon ciliatum

16 Stigmaphyllon lancifolium

12 Stigmaphyllon sagraeanum

10 Thunbergia grandiflora

10, Thunbergia grandiflora var. alba

s- Thunbergia laurifolia

15 - Tristellateia australasica

PALMS

Acoelorraphe Wrightii (S.)

Actinorhytis calapparia

Archontophoenix Alexandrae

Areca catechu-Betel-nut Palm

Arenga saccharifera-Kabong, Sugar Palm

4 Arenga undulatifolia (S.) ..

Carludovica palmata-Panama Hat Palm (Cyclanthaceae)

Chrysalidocarpus lutescens

20 Cyrtostachys lakka-Sealing Wax Palm

Elaeis guineensis-African Oil Palm-(for ornamen

Heterospathe salomonensis (S.)

s. - Licuala grandis

Licuala spinosa

- Livistona chinensis

Livistona cochin-chinensis .

to Livistona rotundifolia

10 Livistona Woodfordii

Oncosperma tigillaria-Nibong

tubers

025

.. 020

100

- 060

.. 0025

.. 040

.. 025

.. 005

.. 025

.. 040

.. 025

.. 025

. 100

. 040

.. 040

.. 040

$\ldots 025$

.. 040

.. 025

1025

.. 025

.. 025

040 .

100

025

025

025 o.

.. 025

.. 025

. 025

Oreodoxa oleracea-Cabbage Palm

Oreodoxa regia-Royal Palm

10 Pinanga Kuhlii

2 Pinanga patula

20. Ptychosperma McArthur

- Ptychosperma sanderiana

Rhapis flabelliformis-suckers, not in pots

10 Rhopaloblaste hexandra (S.)

to Stevensonia grandifolia

6 Verschaffeltia splendida

.

$\cdots$

$\begin{array}{llll}0 & 40 & \end{array}$

.. $040 \gamma$

040 of

. 0404

. 0407

. 040

.. 040

. 040

.. 040

. 040 as

. 040

. 040

. 040

... 040

.. 020

. 040

.. 075

. 075

\section{HERBACEOUS PLANTS AND DWARF SHRUBS}

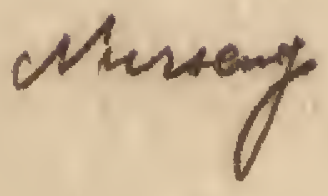
Ageratum, dwarf
Alternanthera paronichioides
Angelonia salicariaefolia
Artemisia lactiflora
- Aster bellidioides
- Aster sp. (dwarf)
4 Aster, perennial-Michaelmas Daisy
Asystasia intrusa
- Barleria cristata, four varieties
Barleria lupulina
Begonia-bedding variety .
- Beloperone guttata
- Canna, hybrids, various races, roots
is. Cestrum nocturnum

$\cdots$
$\cdots$
$\cdots$
$\cdots$
$\cdots$
$\cdots$
$\cdots$


HERBACEOUS PLANTS AND DWARF SHRUBS.-continued

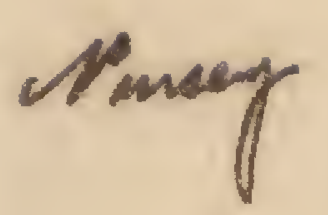

- Clerodendron paniculatum-Pagoda Flower

Coleus Blumei, various races

Coreopsis grandiflora (perennial) ..

Crinum asiaticum-Crinum Lily ..

Crinum giganteum-Java Lilly

- Crossandra nilotica (P.)

- Crossandra undulifolia-Singapore Geranium

- Crossandra undulifolia-yellow variety

- Cuphea platycentra

Cuphea sp. (red)

Daedalacanthus nervosus.

. .

Eranthemum atropurpureum

Eranthemum hypocrateriforme

Eranthemum reticulatum

2 Eranthemum Wattii

Eucharis grandiflora-Amazon Lily ..

* Galphimia glauca

Hedychium coronarium, suckers

- Helianthus angustifolius-Perennial Sunflower Heliconia psittacorum-"Japanese Canna"

* Hippeastrum

Hymenocallis littoralis-Spider Lily ..

Lantana sellowiana

- Mirabilis jalapa-Marvel of Peru

- Orthosiphon stamineus-Cat's Whiskers

- Pancratium zeylanicum

- Pennisetum macrostachyum (S.)

Pentas alba

Pentas carnea

- Pentas coccinea.

Platycodon grandiflorum

Plumbago capensis-Blue Plumbago

Plumbago rosea-Pink Plumbago

Rudbeckia Newmanii-Black-Eyed Susan

Ruellia macrophylla

Ruellia malacosperma

Russellia juncea

Russellia hybrid

Russellia sarmentosa

Sanchezia nobilis

Turnera ulmifolia

Turnera trioniflora

Tussacia pulchella

Verbena tenera.

- Vinca rosea-Periwinkle

Wedelia biflora, double

Zephyranthes, white, yellow and pink

Zephyranthes, hybrids and large flowered forms

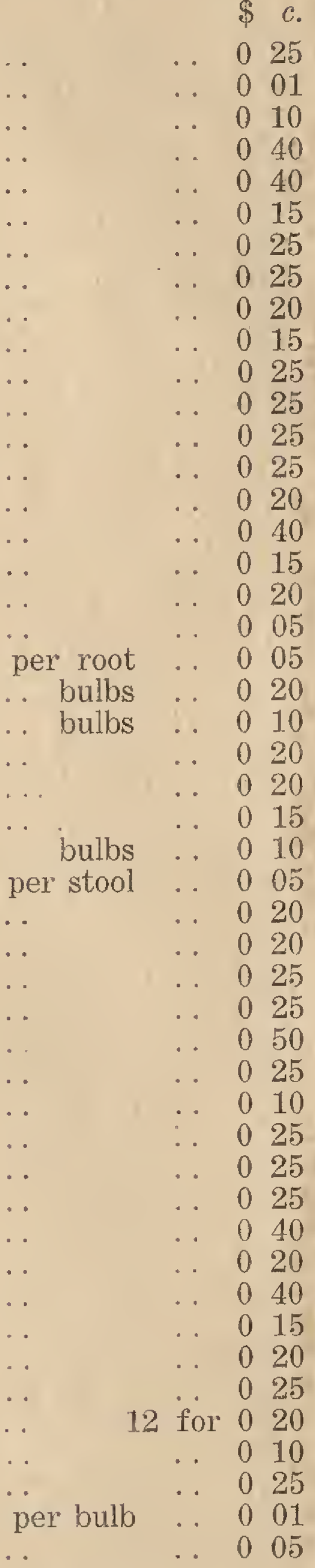

\section{SEEDS OF HERBACEOUS PLANTS}

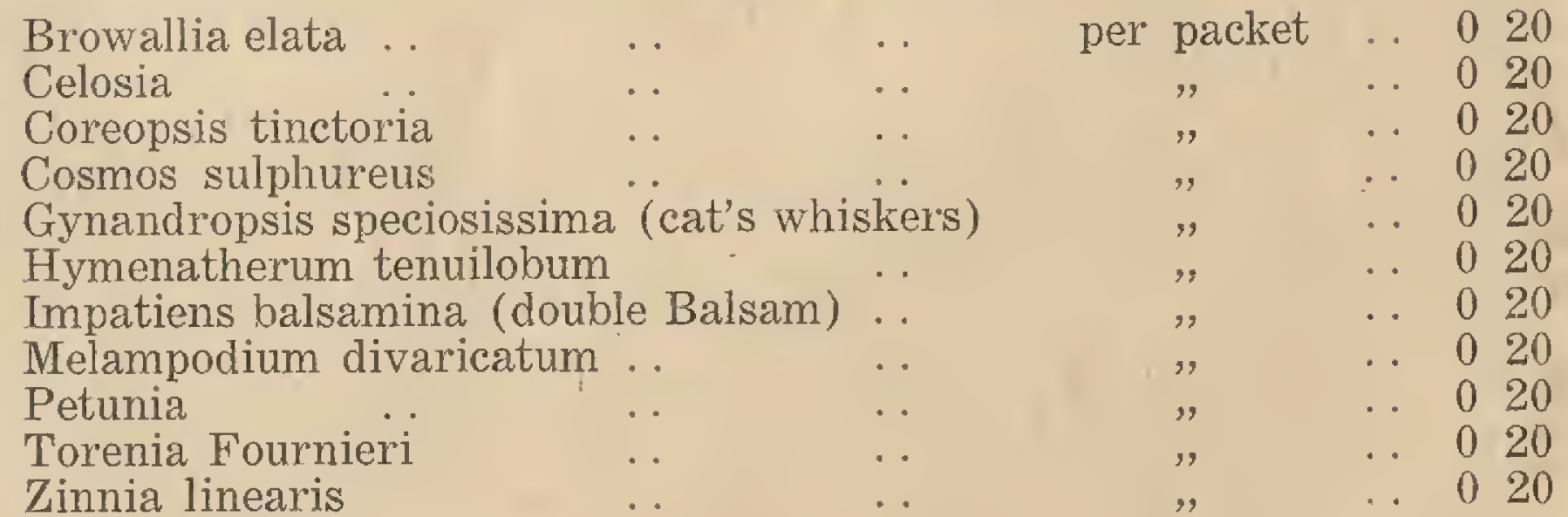

\section{WATER GARDEN PLANTS}

Acorus calamus-Sweet Flag (S.) . . $\quad \ldots .0025$

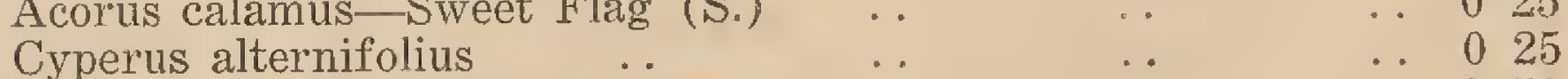

Cyperus papyrus-Nile Papyrus _.. $\quad \ldots \quad \ldots 000$

Eichhornia crassipes-Water Hyacinth $\quad 010$

Hydrocleis nymphoides-Water Poppy $\quad \ldots \quad \ldots 025$

Limnocharis Plumieri (S.) .. . . . 025

$\begin{array}{lllllll}\text { Monochorea elata } & \ldots & \ldots & \ldots & \ldots & 0 & 25\end{array}$

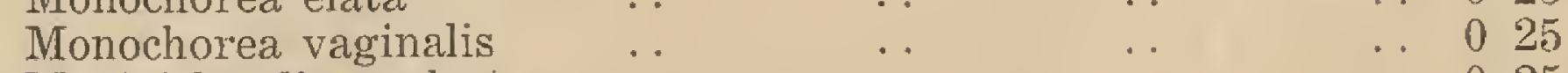

Montrichardia aculeata ... 025

Nelumbium speciosum-Indian Lotus .. $\quad$. . 025

Nymphaea in variety-Water Lilies .. $\quad$ young plants .. 040

Pontederia sp. .. $\quad \ldots \quad \ldots \quad \ldots \quad \ldots 25$

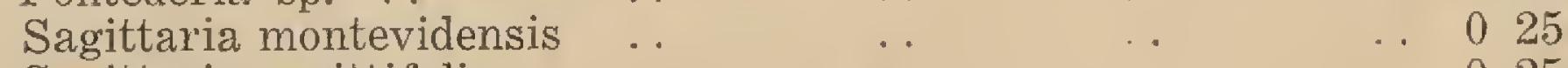

$\begin{array}{lllllll}\text { Sagittaria sagittifolia } & \ldots & \ldots & \ldots & \ldots & 025\end{array}$ 


\section{ORCHIDS}

Arachnis flos-aeris-Scorpion Orchid

$\$ c$.

Arachnis Hookeriana (S.) - White Scorpion Orchid

Arachnis Maingayi-Scorpion Orchid

Spathoglottis plicata

cuttings

020

Spathoglottis plicata white .

Vanda Hookeriana

Vanda hybrid Miss Joaquim

Vanda hybrid Josephine

Vanda teres

Vanda teres var. Andersonii

020

" . . 020

$\cdots \quad 0 \quad 40$

060 per cutting .. 050

$\cdots \quad \ldots 005$

$\therefore \quad \ldots 050$

, . . $\quad 050$

050

Occasionally plants of other orchids are for sale; prices on application. :

[No. C.S.O. 768/38]. 
The following is published for general information:-

\section{LIST OF PLANTS}

(4)

which may usually be obtained from the Botanic Gardens at Singapore and Penang.

(S.) and (P.) mean at Singapore or at Penang only.

* Means suitable for hedgé-máking.

† Unrooted cuttings for hedge-making, 100 for $75 \mathrm{cts}$.

Unless stated otherwise, the prices are for small plants in pots and the price may be altered according to the size of the plant. Rooted cuttings dug from the nursery and not in pots are charged at 20 to 40 cents, according to size. The cost of packing and all subsequent charges are extra.

Plants not included in the following lists are sometimes available.

2 Brownea grandiceps (S.).

4o Cassia fistula-Indian Laburnum

5o. Cassia grandis-Pink Cassia

Cassia javanica . .

15. Cassia nodiosa-Pink Cassia

4 Cassia renigera-Burmese Cassia

- Cassia multijuga

5 Cassia siamea-Johar

co Cassia spectabilis

* Citharexylum quadrangulare

Cochlospermum peligiosum-Buttercup Tree

7 Cratoxylon formosum

Cratoxylon ligustrinum

65 Delonix regia-Flame of the Forest 15 Dillenia indica

7o Enterolobium saman-Rain Tree Erythrina indica-Coral Tree (Dadap) "(P.)

70 Jacaranda filicifolia

to Lagerstroemia floribunda

so Lagerstroemia flos-reginae-Rose of India

10 Lagerstroemia Loudonii

35. Mesua ferrea-Ceylon Ironwood

70 Peltophorum pterocarpum-Yellow Flame

25- Pterocarpus indicus-Angsana

12 Saraca declinata (S.)

20 Saraca indica (S.)

8 Saraca taipingensis (S.)

$45^{-}$Spathodea campanulata-African Tulip Tree 14. Wormia suffruticosa

-

025

025

\section{FOLIAGE TREES}

70 Acacia auriculaeformis

3a Adenanthera pavonina-Saga Tree

3o Albizzia falcata-Batai

16 Andira inermis (S.)

zo Andira surinamensis (S.) .

40 Arfeuillea arborescens

25 Calophyllum inophyllum-Penaga Laut

14. Canangium odoratum-Kenanga

30 * Casuarina equisetifolia-Common $\mathrm{Ru}$..

Casuarina rumphiana (S.)

* Casuarina sumatrana

$60 *$ Cinnamomum iners-Wild Cinnamon

30. Dacrydium elatum-Ru Bukit 
17 Elaeocarpus ganitrus

40 Eugenia grandis-Jambu Laut . $\quad \ldots \quad \ldots \quad \ldots \quad \ldots 25$

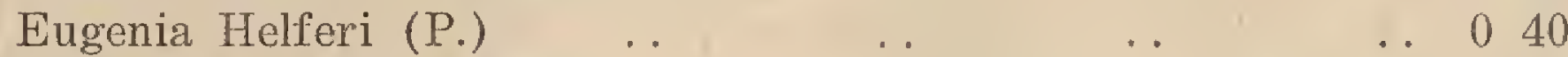

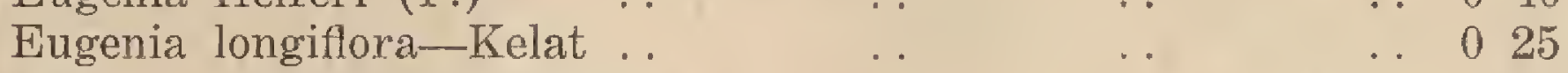

20 Fagraea fragrans-Tembusu $\quad \ldots \quad \ldots . \quad \ldots 025$

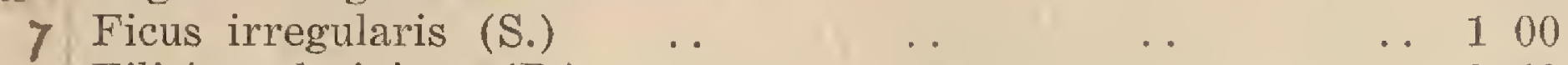

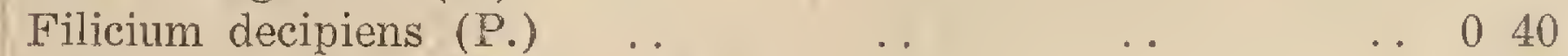
Gliricidia sepium-Mexican Lilac $\quad \ldots \quad \ldots \quad \ldots \quad \ldots 025$ Gnetum Gnemon-Meninjau (P.) . $\quad$. $\quad \ldots 040$

14 Hymenaea courbaril (S.) .. $\quad \ldots \quad \ldots \quad \ldots 025$

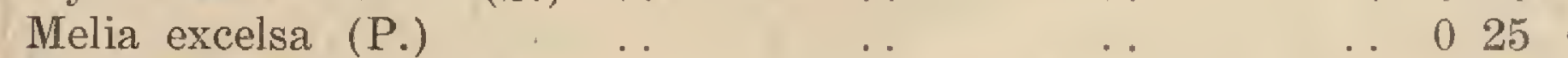

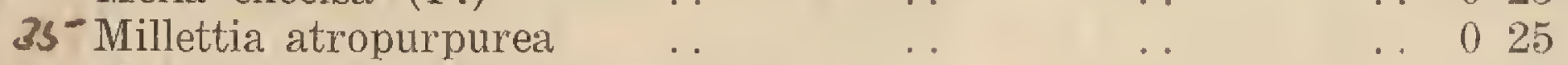

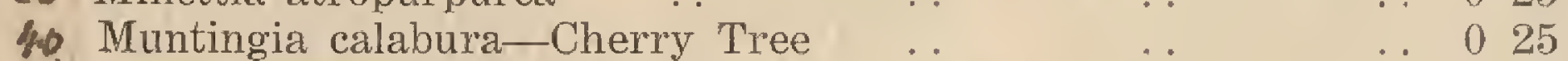
Parkia speciosa (P.)-Petai _.. $\quad \ldots \quad \ldots 040$

$10 *$ Pithecellobium dulce-Madras Thorn .. $\quad \ldots 025$

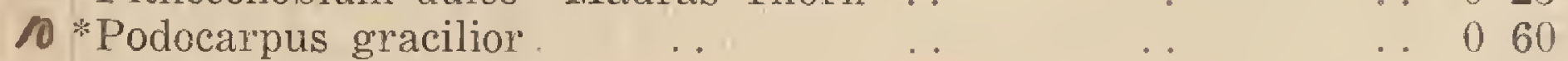

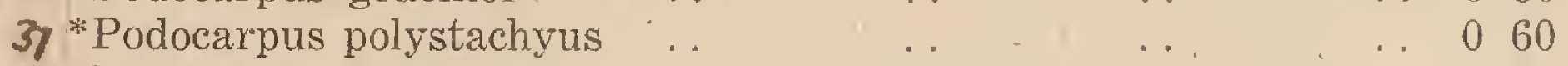

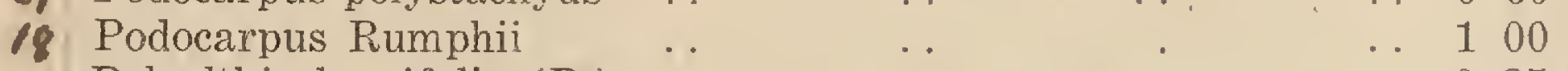

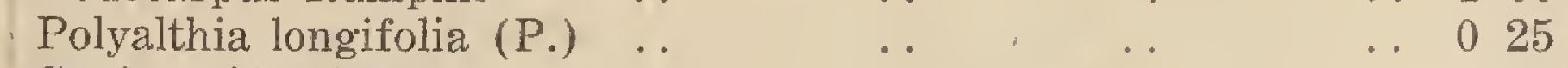

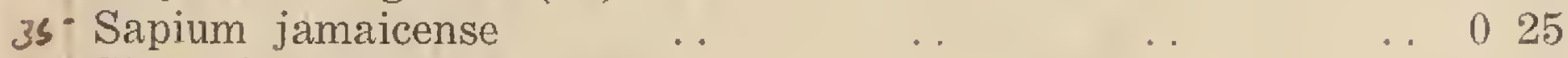

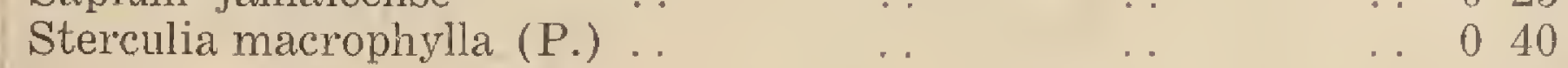

*Streblus asper-per cutting _. $\quad \ldots \quad \ldots 001$

20 Swietenia macrophylla-Broad leaved Mahogany .. $\quad \ldots .040$

30 Tamarindus indica-Tamarind $\quad \ldots \quad \ldots \quad \ldots \quad \ldots 040$

15. Terminalia catappa-Bengal Almond .. $\quad \ldots . \quad \ldots 040$

\section{SHRUBS}

†*Acalypha siamensis (P.)

from nursery bed

o. Bauhinia acuminata (white) Bauhinia tomentosa

*Bixa orellana-Anatto (Kesumba) from nursery bed

30 Bougainvillea Calcutta No. 2 (S.)

025

005

12 Bougainvillea Calcutta No. 13 (S.)

050

050

24 Bougainvillea Cypheri (S.)

10 Bougainvillea formosa

20 Bougainvillea glabra, pale rose variety Bougainvillea glabra, var. magnifica

4ot*Bougainvillea glabra, var. Sanderiana

6 Bougainvillea lateritia

14 *Bougainvillea Mrs. Butt ..

ty Bougainvillea Mrs. Fraser ..

Bougainvillea Mrs. Lancaster

25*Bougainvillea Mrs. McLean ..

25*Bougainvillea Rosa Catalina

10 Bougainvillea Thomasii

10 Brunfelsia americana

10 Brunfelsia eximia (blue)

10 Brunfelsia undulata (white, large flowers)

14 Caesalpinia pulcherrima--Peacock Flower

Caesalpinia pulcherrima var. lutea

6 Calliandra haematocephala (P.)

Cassia auriculata

5 Cassia bicapsularis

6 Cassia biflora

22 Cassia fruticosa

18 Cassia splendida

8 Clerodendron macrosiphon

\& Clerodendron ugandense (S.)

*Crotons (Codiaeum) - several races

20 Cyanophyllum magnificum

6 *Duranta Ellisii

$14{ }^{*}$ Duranta repens

$\uparrow$ Ehretia microphylla_-per cutting

8 Eranthemum malaccense

14 Ervatamia divaricata-Susun Kelapa .

22 Euphorbia pulcherrima--Poinsettia

5." Excoecaria bicolor 
SHRUBS.-continued

s-Gardenia florida-Bunga China

4 Gustavia gracillima

15- Gustavia speciosa

6 Hamelia patens (S.)

-. 060

$\begin{array}{lll}\cdots & 0 & 25 \\ \cdots & 0 & 25\end{array}$

4 Hibiscus mutabilis

of* Hibiscus rosa-sinensis, various races, rooted cuttings

\& Hibiscus, standard plants . .

* *Hibiscus schizopetalus

H Hibiscus sp. (small pink flowers)

2y Holmskioldia sanguinea ..

6 Honckenya ficifolia ..

4 Ipomoea carnea

'3-Ixora chinensis

s- Ixora coccinea and varieties

5 Ixora congesta

10 Ixora javanica and varieties

4. Jasminum bifarium

16 Jasminum glandulosum

9 Jasminum pubescens

16 Jasminum sambac

4 Jatropha multifida

Jatropha pandurifolia and varieties

5- Kopsia fruticosa

8 Lagerstroemia indica (P.) ..

30 Lantana camara, varieties

9 Malpighia coccigera

") * Malpighia nitida

5 - Malvaviscus arborea (S.)

15- Malvaviscus Conzattii

4 Manihot utilissima variegata-Variegated Tapioca

\&s-Murraya paniculata-Kemuning

13 Mussaenda erythrophylla ..

10 Mussaenda luteola

Nerium oleander-Oleander

16 Ochna pumila (S.)

Panax Guilfoylei

Panax fruticosum

10 Petrea rugosa (P.)

10 Phaleria Blumei

18 Plumeria acutifolia-Frangipanni

27 Plumeria obtusifolia

so Plumeria rubra (Red Frangipanni) and varieties.

7 Quassia amara-Bitter Quassia

16. Randia macrantha-Angels Trumpets .

12 *Ravenia spectabilis (S.)

is Rondeletia odorata

4 Sambucus javanica-.Javanese Elder ...

- Sambucus velutina

rolanum Wrighti

to Tecoma Smithii (P.)

2o Tecoma stans-Yellow Bells

15. * Tecomaria capensis

5- Thevetia peruviana-yellow, white and apricot

16 Thunbergia affinis

20 Thunbergia erecta

$\&$ Thunbergia Kirki

Tithonia diversifolia

7 Turnera aurantiaca

10 Uroskinnera spectabilis

25 cents to 050

. 025

. 025

. 025

.. 025

. 025

. 040

. 040

$\therefore \quad 040$

. 040

. 025

.. 025

. 025

.. 025

. 025

.. 025

. 025

. 040

. 0.20

. 025

.. 025

.. 025

.. 025

. 010

. 050

.. 040

.. 025

. 040

. 040

.. 025

.. 025

.. 060

. 040

.. 025

.. 040

.. 040

.. 025

. 025

. 025

060

. 025

. 025

. 025

.. 025

.. 025

. 025

$\therefore \quad 025$

.. 025

025

040

020

. $\quad 025$

040

\section{CLIMBERS}

18 Afgekia sericea

Allamanda cathartica var. Hendersoni .

.

Congea velutina

Cryptostegia madagascariensis (P.) .

.. 040 
7 Faradaya splendida

Ficus pumila (ivy-like creeper) Creeping Fig

Gloriosa superba

Gloriosa virescens

..

$\cdots$

13 Ipomoea digitata-Morning G̈lory (maüe)

10 Ipomoea Horsfalliæ, Briggsii

Ipomoea Leari-Morning Glory (blue)

Ipomoea pulchella, from nursery beds .

7 Jacquemontia violacea

Jasminum rex

10 Lonicera japonica-Japanese Honeysuckle

s- Lonicera macrantha-Indian Honeysuckle

23-Odontadenia speciosa

13 Pandorea jasminoides

10 Pandorea pandorana

«s Pandorea ricasoliana (S.) .

10 Passiflora laurifolia-Buah Susu

15- Passiflora quadrangularis-Grenadilla

16. Passiflora quadriglandulosa

16 ' Petrea volubilis

1) Porana volubilis-Bridal Creeper

6 Quisqualis indica-Drunken Sailor

12 Roupellia grata

2 Stephanotis floribunda

20 Stigmaphyllon ciliatum

i4 Stigmaphyllon lancifolium

10 Stigmaphyllon sagraeanum

16 Thunbergia grandiflora

sy Thunbergia grandiflora var. alba

17 Thunbergia laurifolia

is - Tristellateia australasica

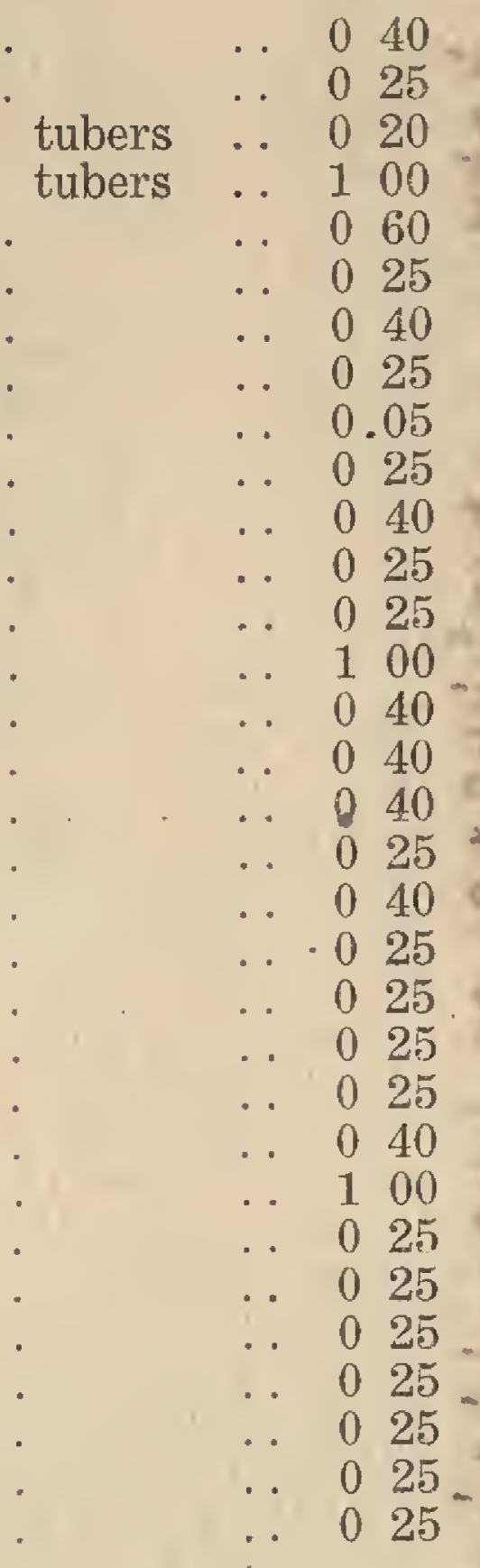

PALMS

3
12
12

Acoelorraphe Wrightii (S.)

Actinorhytis calapparia

Archontophoenix Alexandrae

Areca catechu-Betel-nut Palm

20 Arenga saccharifera-Kabong, Sugar Palm

\$ Arenga undulatifolia (S.) ... Hat Paim (Cyclanthaceae)

7 Chrysalidocarpus lutescens,..

2o Cyrtostachys lakka-Sealing Wax Palm

24. Elaeis guineensis-African Oil Palm (for ornamental purposes)

Heterospathe salomonensis (S.)

40 Licuala grandis

G Iicuala spinosa

s- Livistona chinensis

Livistona cochin-chinensis .

10 Livistona rotundifolia

22 Livistona Woodfordii

30 Oncosperma tigillaria-Nibong

so Oreodoxa oleracea-Cabbage Palm

so Oreodoxa regia-Royal Palm

10 Pinanga Kuhlii

2. Pinanga patula

20 Ptychosperma McArthuri

2o Ptychosperma sanderiana.

Rhapis flabelliformis-suckers, not in pots

7 Rhopaloblaste hexandra (S.)

10 Stevensonia grandifolia

Verschaffeltia splendida

$\therefore \quad 040$

$\begin{array}{lll}0 & 40\end{array}$

$\begin{array}{lll}0 & 40\end{array}$

040

- 040

. 040

100

$\begin{array}{ll}0 & 40\end{array}$

075

040

040

$0 \quad 40$

$\begin{array}{ll}0 & 40\end{array}$

$\begin{array}{lll}0 & 40\end{array}$

$\begin{array}{lll}0 & 40\end{array}$

040

$\begin{array}{ll}0 & 40\end{array}$

$\begin{array}{lll}0 & 40\end{array}$

. 040

. 040

.. 040

. 040

. 040

.. 040

$\therefore 020$

. 040

. 075

.. 075

\section{HERBACEOUS PLANTS AND DWARF SHRUBS}

Ageratum, dwarf

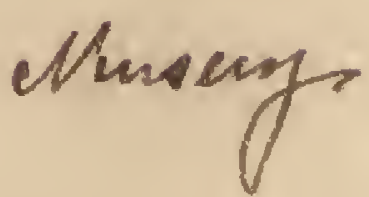

Alternanthera paronichioides

4 Angelonia salicariaefolia

4 Artemisia lactiflora

- Aster bellidioides

¿Aster sp. (dwarf)

- Aster, perennial-Michaelmas Daisy

- Asystasia intrusa

- Barleria cristata, four varieties

- Barleria lupulina

Beronia-beddino variety .

Beloperone guttata

4 Canna, hybrids, various races, roots

10: Cestrum nocturnum

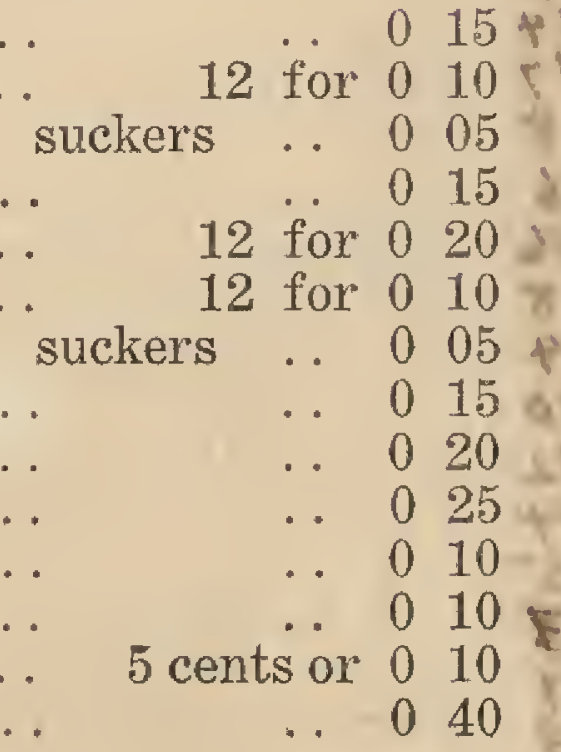


HERBACEOUS PLANTS AND DWARF SHRUBS.-continued

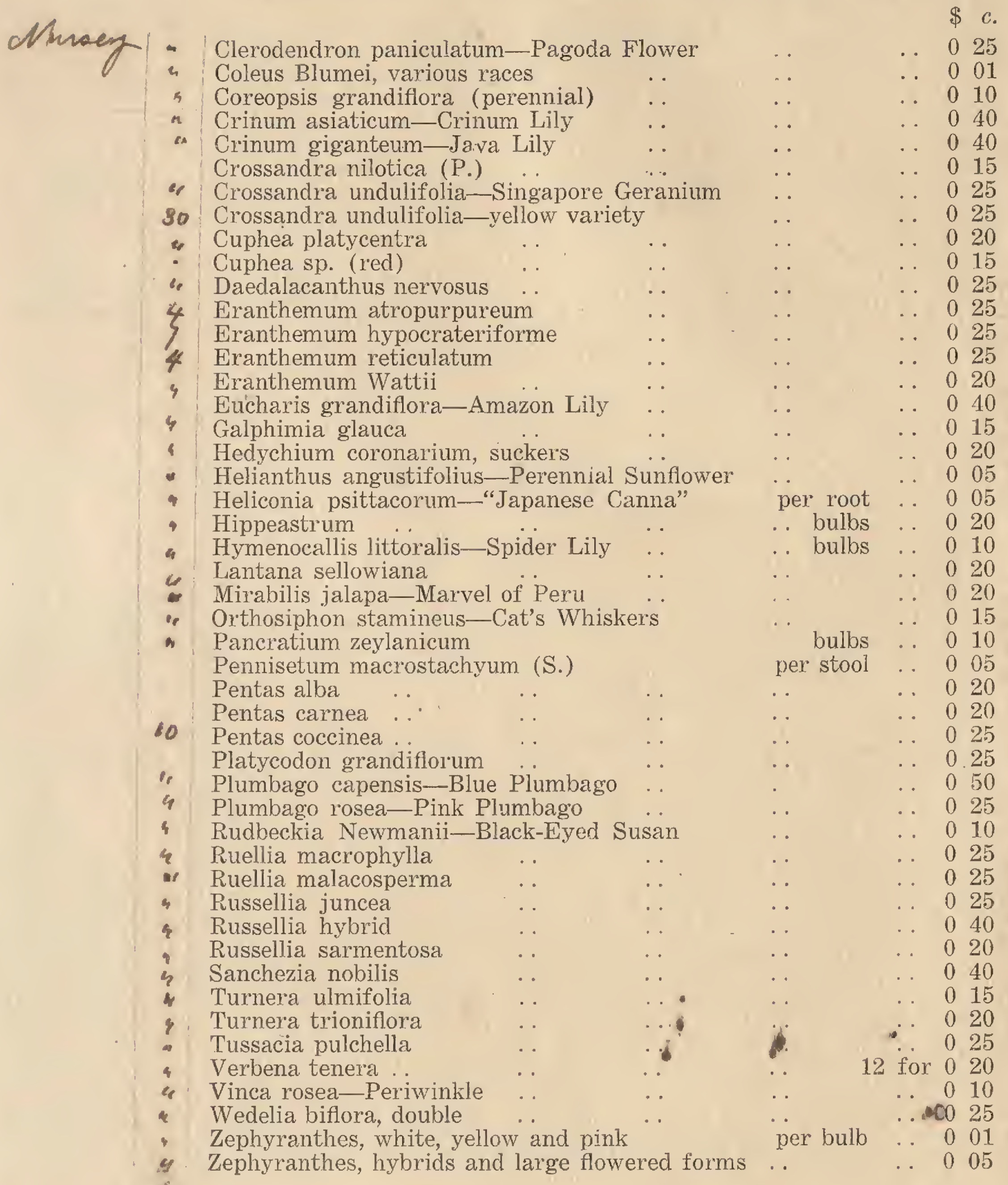

\section{SEEDS OF HERBACEOUS PLANTS}

Browallia elata

Celosia

Coreopsis tinctoria

Cosmos sulphureus

Gynandropsis speciosissima (cat's whiskers)

Hymenatherum tenuilobum

Impatiens balsamina (double Balsam) .

Melampodium divaricatum ..

Petunia

Torenia Fournieri

Zinnia linearis

$\begin{array}{ll}\ldots & \ldots \\ \therefore & \ldots \\ \text { (cat's whiskers) } \\ \text { le Balsam) } \\ \ldots \\ \ldots & \cdots \\ \cdots & \cdots \\ \ldots & \cdots\end{array}$

per packet

.. 020

.. 020

. 020

- 020

.. 020

$\therefore \quad 020$

. 020

. 020

. 020

. 020

- 020

\section{WATER GARDEN PLANTS}

Acorus calamus Sweet Flag (S.)

Cyperus alternifolius

(S.)

. $\quad$.

Cyperus papyrus-Nile Papyrus

Eichhornia crassipes-Water Hyacinth

Hydrocleis nymphoides-Water Poppy

Limnocharis Plumieri (S.) .

Monochorea elata

Monochorea vaginalis

Montrichardia aculeata

Nelumbium speciosum-Indian Lotus ..

Nymphaea in variety-Water Lilies .

Pontederia sp. .

Sagittaria montevidensis

Sagittaria sagittifolia

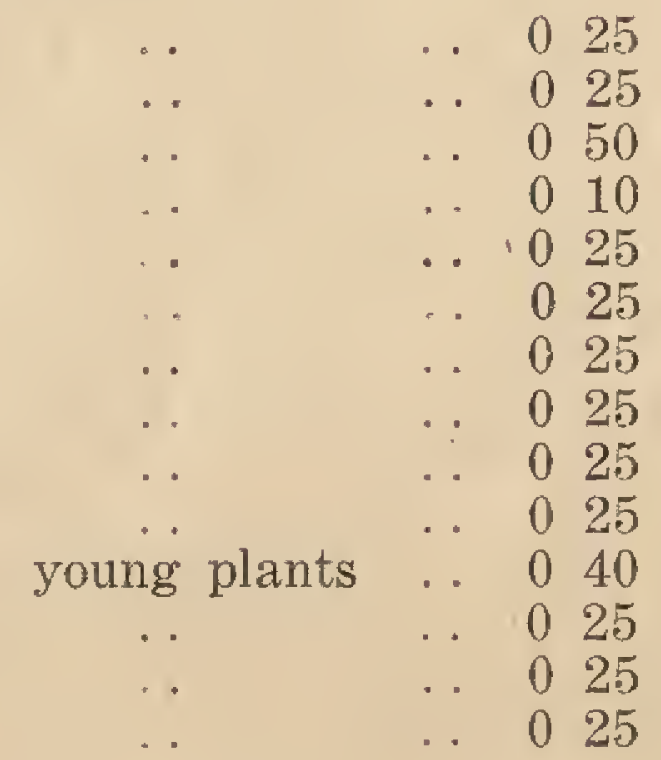


Arachnis flos-aeris-Scorpion Orchid

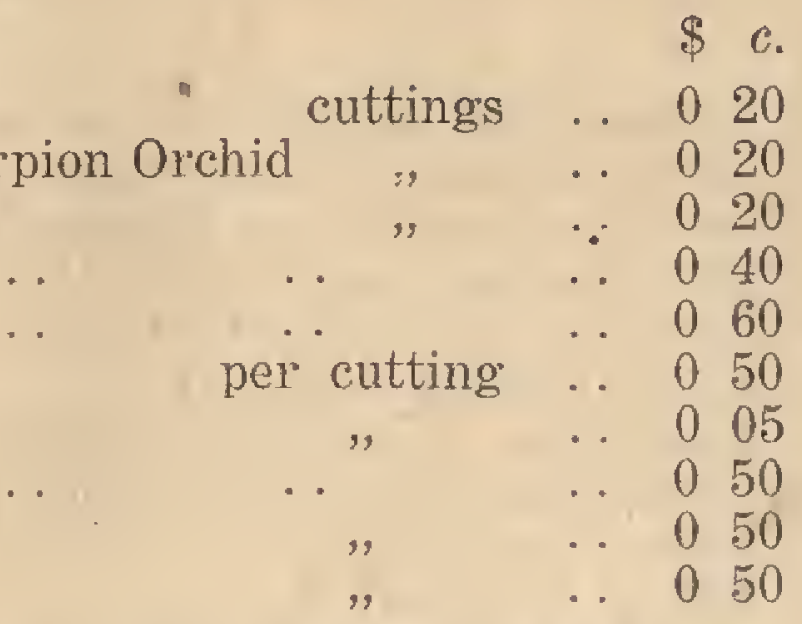

Arachnis Hookeriana (S.) - White Sco

Spathoglottis plicata

Spathoglottis plicata white .

Vanda Hookeriana

Vanda hybrid Miss Joaquim

Vanda hybrid Josephine

Vanda teres

Vanda teres var. Andersonii

. 050

Occasionally plants of other orchids are for sale; prices on application.

[No. C.S.O. 768/38]. 
No Minutes should be written on this page. A separate half-sheet to be used if required. 